\title{
Bracovirus sneaks into apoptotic bodies transmitting immunosuppressive signaling driven by integration-mediated elF5A hypusination
}

Kaijun Luo ( $\sim$ kaijun_luo@ynu.edu.cn )

Yunnan University https://orcid.org/0000-0003-3900-1177

\section{Biological Sciences - Article}

Keywords: Microplitis bicoloratus bracovirus, Immunosuppression, elF5A, Hypusination, Viral integration, Nucleocytoplasmic transport, Apoptotic body, extracellular vesicles, viral reintegration, transmitting immunosuppressive signaling

Posted Date: November 4th, 2021

DOI: https://doi.org/10.21203/rs.3.rs-1049019/v1

License: (a) (i) This work is licensed under a Creative Commons Attribution 4.0 International License.

Read Full License 


\section{Abstract}

The typical characteristic of polydnavirus (PDV) infection is persistent immunosuppression, governed by the viral integration and expression of virulence genes. Recently, how Microplitis bicoloratus bracovirus (MbBV) activates caspase-3 to cleave Innexins, gap junction proteins, has been highlighted, further promoting apoptotic cell disassembly and apoptotic body (AB) formation. However, little is known about how inserted viral fragments interact with host cells to drive apoptosis and the role MbBV-mediated extracellular vesicles play in immune suppression. Herein, we show that ABs transmitted immunosuppressive signaling, causing recipient cell apoptosis and dismigration. Overall, viral host integrated motif sites insertion damaged host genome stimulating elF5A nucleocytoplasmic transport, activating the elF5A-hypusination translation pathway. Functionally, translated apoptosis-related host proteins, such as P53, CypD, CypJ, and CypA, drive a broken dsDNA cellular apoptosis. Furthermore, translated viral proteins, vank86, 92, and 101, known to complex with transcription factor Dip3, positively regulated DHYS and DOHH transcription maintaining the activation of the elF5A-hypusination translation pathway. Mechanistically, MbBV-mediated extracellular vesicles contained inserted viral fragments that re-integrated into recipients, potentially via the homologous recombinant repair system. Meanwhile, this stimulation regulated activated caspase-3 level via pl3K/Akt 308 and 473 dephosphorylation to promote recipients cell apoptosis; pl3K/Akt 473 phosphorylation inhibited caspase-3 activation leading to recipient cell dismigration. In conclusion, our results suggest that integration-mediated elF5A hypusination drives extracellular vesicles for continues immunosuppressive.

\section{In Brief}

Zhou et al. demonstrated that bracovirus invades apoptotic bodies where integration-mediated elF5A hypusination drives immunosuppressive signaling transmission to recipients, which have different immunosuppressive characteristics, such as dismigration and apoptosis. Reintegration of apoptotic bodies' Bracovirus fragments affects different Akt phosphorylation sites, leading to a different immune response of recipient cells.

\section{Highlights}

1. Microplitis bicoloratus bracovirus (MbBV) integration activated the elF5A-HDYS-DHSS hypusination pathway

2. elF5A-hypusination drives immunosuppressive signaling transmission in apoptotic bodies

3. Recipient cells displayed dismigration and apoptosis

4. Reintegration of MbBV in recipient affected pl3K/Akt phosphorylation sites

\section{Introduction}


Immunosuppression and immunosuppressive signaling transmission are important mechanistic elements of immune responses requiring deep exploration. In the virus-host immune system, the virus hijacks the host, hiding in extracellular vesicles (EVs) to escape the immune response, spread into new hosts, and generate progeny viruses. However, whether and how non-replicating viruses, like polydnavirus (PDV), transmit immunosuppressive factors remain unknown.

EVs are vectors for intercellular communication, especially mediating immune responses (Mathieu et al., 2019). Apoptotic bodies (ABs) $(1-5 \mu \mathrm{m})$, the largest type of EVs, are formed by chromatin shrinkage, membrane blebbing, and distribution of cell contents to different vesicles (Kerr et al., 1972; Mathieu et al., 2019). Therefore, $A B s$ are usually rich in various biological substances, including proteins, nucleic acids, and metabolites (Akers et al., 2013; Ratajczak et al., 2006; Valadi et al., 2007). ABs can protect these biomolecules, especially RNA and molecules containing genetic information, from degradation and denaturation under different physiological conditions (Taylor and Gercel-Taylor, 2008). The horizontal gene transmission between cells was also confirmed to be efficiently mediated by ABs (Holmgren et al., 2002; Soldan et al., 2002). This unique intercellular communication helps regulate tissue homeostasis. Studies have reported how this intercellular communication can also be used by viruses (Batagov and Kurochkin, 2013; Valadi et al., 2007; Vojtech et al., 2014). EVs isolated from human hepatoma cells infected with hepatitis $\mathrm{C}$ virus (HCV) contain full-length viral RNA, viral proteins, and even viral particles (Ramakrishnaiah et al., 2013). Interestingly, EVs containing HCV RNA are not easily affected by antibodies (Ramakrishnaiah et al., 2013). Therefore, using EVs for virus transmission is an effective immune escape strategy. In addition, the virus can exploit EVs to regulate the immune system (Canitano et al., 2013; Meckes et al., 2013; Pegtel et al., 2010). The latent membrane protein-1 (LMP1) of human herpesvirus has been found in EVs and can effectively inhibit the immune response (Dukers et al., 2000; Flanagan et al., 2003; Keryer-Bibens et al., 2006). Similarly, Herpes simplex virus can deliver interferon gene stimulators, viral RNAs, and mRNAs to uninfected cells by transmitting them through EVs (Kalamvoki et al., 2014). Of course, EVs been endowed with these functions are related to primary infective host.

Viral integration into the host genome trigger immune response. The goal of viral integration is to transcribe viral genes and translate viral proteins. Of note, a damaged host genome stimulates an immune response (Chatzinikolaou et al., 2014). Nevertheless, the most controversial observation is that viruses inhibit the host translation system while needing to translate viral proteins. How viruses solve this issue is an interesting question.

Eukaryotic translation initiation factor 5A (elF5A) plays an important role in modulating viral replication. Human immunodeficiency virus (HIV) was the first virus suggested to require elF5A through regulating the expression of virion protein (Rev)-dependent nucleocytoplasmic transport on elF5A (Elfgang et al., 1999; Hoque et al., 2009; Olsen and Connor, 2017; Suhasini and Reddy, 2009). EBOV also requires elF5A to regulate the secondary transcription of EBOV genes by modulating the translation of the transcription factor VP30 (Olsen and Connor, 2017; Olsen et al., 2018). During translation elongation, the chemical characteristics of the acceptor and donor amino acids can affect peptide bond formation and slow down 
the elongation process (Gamble et al., 2016; Gardin et al., 2014; Sabi and Tuller, 2015). Considerable effects in ribosome pausing have been explicitly described for proline and positively charged amino acids (Artieri and Fraser, 2014; Pavlov et al., 2009; Wohlgemuth et al., 2008). Studies have supported ribosome stalling in the absence of elF5A when proline, lysine/arginine, glycine, or aspartic/glutamic is/are immediately upstream of the aminoacyl tRNA at the P-site (position-11), and when acidic to basic amino acids are two positions upstream of the P-site (position-8) (Pelechano and Alepuz, 2017).

Hypusine is formed by conjugation of the aminobutyl moiety of spermidine to a specific lysine residue of elF5A. elF5A isoforms is a single family of cellular proteins modified by the natural acid hypusine ( $\mathrm{N} \varepsilon-4$ amino-2-hydroxybutyl (lysine) derived from the polyamine spermidine (Park and Wolff, 2018). The posttranslational modification of hypusine involves two enzymatic steps (Park et al., 2006), catalyzed by deoxyhypusine synthase (DHPS) (Joe et al., 1995; Wolff et al., 1995) and deoxyhypusine hydroxylase (DOHH) (Abbruzzese et al., 1986; Park, 2006; Park et al., 1981). Hypusine is essential for elF5A activity; inactivation of genes encoding either elF5A or DHPS is lethal in yeast and mouse (Park and Wolff, 2018), underscoring the vital role of elF5A hypusination in eukaryotic cell growth and animal development. However, little is known about the interaction between viral integration and elF5A hypusination.

Due to its small size $(17 \mathrm{kDa})$, elF5A readily leaks through the sieve-like barrier of NPCs into nuclei (Lipowsky et al., 2000), where it loses its cytoplasmic function and possibly also engages in deleterious off-target interactions, such as non-specific RNA binding or competition with the ribosome export-adapter Nmd3 (Malyutin et al., 2017). The mammalian exportin Xpo4 captures mislocalized nuclear elF5A and retrieves it back to the cytoplasm (Lipowsky et al., 2000). Strong evidence suggests that the hypusine modification of elF5A dictates its localization in the cytoplasmic compartment, which is required for protein synthesis (Ishfaq et al., 2012; Lee et al., 2009).

Herein, we attempt to understand how the non-replicating Microplitis bicoloratus bracovirus (MbBV) persistently suppresses the immune system of lepidopteran hosts by leveraging the DNA-delivery capacity of $A B s$. We identified viral gene fragments in $A B s$ induced by MbBV and determined their functional role in immunosuppression in uninfected recipient cells. MbBV, a member of the PDV family, plays an essential role in promoting the growth and development of parasitoid larvae in lepidopteran hosts (Cui et al., 2019; Luo et al., 2007). Although MbBV loses the ability to replicate in lepidopteran hosts due to its unique replication mechanism, MbBV-mediated immunosuppression of lepidopteran hosts can persist (Bezier et al., 2009; Burke and Strand, 2012; Gundersen-Rindal et al., 2013; Wetterwald et al., 2010). Immunosuppression is usually caused by viral gene integration into the host cell genome to perform transcription, such as that of vanks and PTPs (Yu et al., 2016). Cellular immunity is the most critical defense against parasites in Lepidoptera (Stanley et al., 2009); however, the transcription products of viral genes can damage the system by inducing apoptosis and cytoskeletal depolymerization in hemocytes (Beck and Strand, 2005; Cai et al., 2021; Eum et al., 2010; Gitau et al., 2007; Kumar and Kim, 2016; Labropoulou et al., 2008; Luo and Pang, 2006a; Pruijssers and Strand, 2007; Thoetkiattikul et al., 2005). Furthermore, in our previous studies, we found that inhibiting the PI3K/Akt pathway induced the 
shutdown of hemichannel, which triggered apoptosis and promoted apoptotic corpuscle formation (Chen et al., 2021).

In this study, we address two major gaps in understanding the mechanism underlaying immunosuppression: (1) what is the driver of apoptosis, and (2) how can ABs transmit immunosuppressive signaling?

\section{Results}

Bracovirus mediates the formation of $A B s$ that transmit immunosuppressive signaling

We have previously shown that MbBV induces apoptotic body formation by shutting down hemocyte hemichannels to activate caspase-3 and cleave inxs, triggering apoptotic cell disassembly (Chen et al., 2021). However, the biological role of MbBV-induced ABs in immunosuppression remained poorly understood until the present study (Figure 1A). Here, we constructed an N-terminal elongated recombinant baculovirus, reBac-His-TEV-Inx3 (reBac-Inx3), capable of delivering virus particles through ABs as a positive control to investigate whether MbBV suppresses the immune response via $A B$ phagocytosis. The reBac-Inx3 expressed Inx3 with a TEVase cleavage site and 6xHis Tag (Figure S1A), which indirectly induces apoptosis by closing the hemichannel (Chen et al., 2016; Guo et al., 2015) (because of the altered protein structure caused by the extension of the cleavage site and the $6 \times$ His tag at the $\mathrm{N}$-terminal (Chen et al., 2021)). Then, the ABs were collected using gradient centrifugation after Sf9 cells were infected with reBac-Inx3 for $72 \mathrm{~h}$, and were divided equally (Figure S1B). One fraction was used for direct protein quantification from the $A B s$, and the other was incubated with $S f 9$ cells for $72 \mathrm{~h}$ before cell protein collection. We observed a significant increase in the Sf9 cells by comparing the $6 \times$ His tag expression in $A B s$ and Sf9 cells incubated with ABs (Figure 1B). However, the $6 \times$ His tag expression was significantly higher in Sf9 cells incubated with equal ABs (Figure 1C); in contrast, it was significantly lower in ABsincubated Sf9 cells than in reBac-Inx3-infected Sf9 cells (Figure 1D). Thus, the overexpression of $6 \times$ His tags in Sf9 cells after incubation with ABs supports the position that reBac-Inx3-induced ABs can act as viral vectors.

To further confirm the biological role of ABs in recipient cells, we designed Abs of UV-irradiated Sf9 cells without biological and chemical pollution (Chen et al., 2015; Mohan, 2003) as a negative control. Accordingly, we treated Sf9 cells using different apoptosis induction methods to promote apoptotic body formation (Figure 1E) and collected ABs using gradient centrifugation (Figure S1C). Expectedly, the total number of apoptotic cells incubated with different concentrations of UV-induced ABs was not significant (Figure 1F; Figure S1D). The ABs induced by MbBV promoted Sf9 cell apoptosis when the concentration exceeded $30 \%$ (Figure $1 \mathrm{G}$ ). Similarly, apoptosis of Sf9 cells was significantly increased by ABs induced by reBac-Inx3 (Figure 1H). We also detected the effect of different ABs on cell numbers and found no significant differences in UV- and MbBV-induced ABs (Figures $1 \mathrm{I}$ and $1 \mathrm{~J}$ ). In contrast, a significant decrease in the cell number was observed using ABs induced by $5 \%$ reBac-TEV-Inx3 (Figure 1K). These results show that the MbBV-induced $\mathrm{ABs}$ can promote the apoptosis of recipient $\mathrm{Sf} 9$ cells. 
Since the disturbance of dynamic cytoskeleton regulation is one of the crucial phenotypes of bracovirus infection (Gitau et al., 2007; Kumar and Kim, 2016; Labropoulou et al., 2008; Luo and Pang, 2006a), ABs induced by different apoptotic induction methods were incubated with scratch High Five cells in a concentration gradient manner for $24 \mathrm{~h}$ (Figure 1L). ABs induced by UV irradiation did not affect the wound healing rate of High Five cells with the increase in concentration (Figures 1M, and 1P). In contrast, ABs induced by MbBV and reBac-Inx3 inhibited the migration of High Five cells at higher concentrations, MbBV $60 \%$ (Figure 1N) and reBac-Inx3 from 10-60\% (Figure 10). Results confirmed that MbBV-induced ABs could inhibit the dynamic cytoskeleton regulation in High Five cells.

However to determine wonder what endowed these functions of $A B s$, we investigated how MbBV regulates primary infected cells, and how are viral proteins are translated.

Bracovirus integrates host $S$. litura genome via HIM

Microplitis demolitor bracovirus (MdBV) (Beck et al., 2011) and Cotesia congregata bracovirus (CcBV) (Chevignon et al., 2018), both of which have HIM, are polydnaviruses well known to integrate into the host genome (Figures S2A-S2C). The integrated genome of $S$. litura cells was sequenced to scan integrated viral fragments. Natural parasitized hemocytes and MbBV-infected Spli221 cells were sequenced (Figure 2A). The MbBV genome compared with MdBV and CcBV genomes to identify the HIM and wasp integrated motif of MbBV; compared with bracovirus-integrated $S$. litura DNA sequence to isolate HIM-containing fragments. Finally, by mapping fragments to $31 \mathrm{~S}$. litura chromosomes (Cheng et al., 2017), the position of integrated MbBV DNA was identified and quantified (Figure S2D). Viral integrated DNA into host cells were identified using six fragments containing MbBV DNA, Junction 1, and host chromosome (Figure 2B), six fragments containing host DNA, Junction 2, and MbBV DNA (Figure $2 C$ ), which were mapped to the genome of $S$. litura (Figure 2D). Overall, viral DNA did not integrate into all host chromosomes; integrated MbBV DNA was found only in 21 of 31 chromosomes. The remaining ten chromosomes showed no viral integration, including Chr 3, 8, 11, 13, 21, 28, 30, and Z.

The structure of MbBV HIMs was predicted, and sequences containing the HIM junction were selected (Table S1). All 17 HIMs had similar structures, including two homologous boundary sites (GAAAATTTC) on both the $5 \quad$ terminals, one homologous junction 1 (CTAGT), one homologous junction 2 (ACTAGG), and a non-homologous loop (Figure S2E). These two junctions were used when MbBV DNA integration occurred in different scaffolds (Figures S2F and S2G; Table S2). On measuring the quantity of these scaffolds and HIMs, we found that most scaffolds appeared once, except for JS774326 that appeared 15 times. JS945511 appeared four times, and JS399327 twice. Similarly, most HIM appeared once, except HIM 4 that appeared twice; HIM 14, 7 times; HIM 16, 21 times; and HIM F157, 22 times. Primers were designed to amplify HIM-C11 in Chr 1, HIM-C14 in Chr 9, HIM-C10 in Chr16, and HIMC16 in Chr 19 (Figure 2E), in both parasite hemocytes and MbBV-infected Spli221 cells to further confirm the above results (Figures 2F and 2G). Compared with the MbBV genome (Yu et al., 2016), 7 of 17 circles were integrated, including viral circular (contained genes): C4 (ptp66), C10 (vank92), C11 (ptp107 110, 
112, 113, and vank111), C14 (ptp77, 72, 78, and vank86), C15 (HP114,115), C16 (ptp88, 61, 87, 66, 64, Vank89, 99, 100, 101, 102), F157(vank76) (Figure 2D). Vanks 86, 99, 100, 101, ptp 102 vank76 were detected in host hemocytes (Figure $2 \mathrm{H}$ ).

In conclusion, the MbBV-integrated genome in the host cells via $17 \mathrm{HIM}$ in 21 host chromosomes; at least 7 of $17 \mathrm{MbBV}$ circles contained HIM sites and integrated into the host chromosomes.

\section{Bracovirus integration triggers elF5A hypusination}

Previously, we found that MbBV inhibits the elF4E-elF4A axis (Cui et al., 2019) and its regulated mechanism by which viral protein translates (Cai et al., 2021). We speculated that there exists another translation pathway that may rescue protein translation. Hemocytes were collected to detect the elF5ADHYS-DOHH pathway, showing that elF5A was hypusinated and DHYS and DOHH expression was upregulated 6 days post parasitism (Figures 3A and 3B). To further confirm that MbBV activates the elF5A hypusination pathway, purified MbBV was used to infect High Five cells. elF5A hypusination, DHYS, and $\mathrm{DOHH}$ levels presented a dose-dependent relationship (Figures S3A-S3E). We also confirmed that MbBV activated the elF5A-DHYS-DOHH protein translation pathway.

Next, we performed transcriptomic and proteomic analyses to identify the translated proteins of $S$. litua hemocytes triggered by elF5A hypusination under natural parasitization. iTRAQ was performed using hemocytes, and MS/MS data were analyzed with transcription mRNA data (Li et al., 2014) together, from two proteomes, M and S (M: Parasitized hemocytes; S: Non-parasitized hemocytes) and three transcriptomes, All, M, and S (All: Mixture, M and S as above) (Figures S4A-S4C), a total of 1853 proteins and 8625 mRNAs were separated into three classifications: continuous expression 5522 mRNAs (being found in the three pools, All, M, and S) and 1604 proteins (in the two pools M and S); inducible 755 mRNAs (in All and M) and silenced 1575 mRNAs (in All and S) (Figure 3C). All of these proteins were enriched in 20 KEGG pathways (Figure S4D). First, we found forty-six proline-rich proteins containing PPP and PPG, which were reported as a hallmark of elF5A hypusination translation proteins, only eight prolinerich proteins, HSP73X2, PUR6, IF2B1X2, ACLYX2, VATPA, TPA3, PPN were upregulated; and comparing with their mRNAs presented in a heatmap (Figure S5A). These results suggested that the mechanisms underlying MbBV-mediated elF5A-hypusination and protein translation might expand current knowledge.

Secondly, based on previous data showing that ribosomes are stalled in the absence of hypusinatedelF5A when proline $(P)$ and glycine $(G)$, or charged amino acids [aspartic acid $\left(D^{-}\right)$, glutamic acid $\left(E^{-}\right)$, lysine $\left(\mathrm{K}^{+}\right)$, arginine $\left(\mathrm{R}^{+}\right)$, and histidine $\left(\mathrm{H}^{+}\right)$] are located upstream of the P-aminocarbonyl tRNA (Pelechano and Alepuz, 2017), we further identified the involved proteins. Our analysis revealed that all upregulated proteins were involved in 10 pathways (Figure 3D). Over 35\% of 7 amino acids (P, G, D, E, K, R) were separated (Figure S5B), and 10 proteins were highly expressed and used in this study as hallmarks of hypusination (Table S3). We also found that the cellular apoptosis-related proteins, P53, CypD, CypJ, and CypA, were highly expressed under hypusination 6 days post parasitism (Figure 3E and 
3F). These results showed that conserved K52 was modified by hypusination in S. litura, and its vicinity was highly conserved from yeast to humans (Park et al., 2010; Schuller et al., 2017)(Figure 3G).

elF5A is hypusinated through a two-enzyme cascade, in which an aminobutyl group from the polyamine spermidine is first covalently attached to lysine 52 of elF5A by deoxyhypusine synthase (DHYS) and then hydroxylated by deoxyhypusine hydroxylase (DOHH) (Shin et al., 2017). Moreover, MbBV upregulated DHYS and DOHH expression (Figure $3 \mathrm{H}$ ). In conclusion, these results demonstrated that natural parasitization of $M$. bicoloratus promoted hemocyte hypusination of elF5A and triggered hypusination-dependent protein translation by blocking elF4E-elF4A translation during immunosuppression of S. litura.

Bracovirus integrated viral genes dependent elF5A hypusination translation pattern and viral proteins positively regulated DHYS and DOHH expression

MbBV-integrated genes were highly expressed following hypusination. GC7 inhibited DHYS and blocked the elF5A hypusine signaling pathway (Figure S6A). Experimental results showed that GC7 inhibited the hypusine modification of elF5A in a time- and concentration-dependent manner (Figures 4A4D). Inhibition of ectopic expression of the V5-fusion proteins in the High Five cells using $10 \mu \mathrm{M} \mathrm{GC7} \mathrm{for}$ $72 \mathrm{~h}$, viral Ank proteins were inhibited by DHYS inhibition GC7, presenting the same pattern as hypusinedependent proteins, namely P53, CypD, CypA, and CypJ (Figures 4E-4R). The data above demonstrate that $\mathrm{MbBV}$ regulates the translation of seven host and viral proteins rich in proline, glycine, and amino acids through hypusine (Table S3). Previous research has shown that vank-Dip3 inhibits the transcription of elF4E-elF4A and its regulated genes (Cai et al., 2021); however, whether and how Dip3 regulates DHYS and $\mathrm{DOHH}$ during viral Ank protein expression are not well understood. Accordingly, we performed RTqPCR to determine how Dip3 regulates the transcription of DHYS- and DOHH-encoding genes. MbBV promoted the transcription of DHYS and DOHH, which was not predicted to be inhibited; then overexpression Dip3 inhibited the transcription of DHYS and DOHH (Figures 4S and 4T). Next, to determine whether Dip3 indeed inhibited the transcription of DHYS and DOHH, we designed RNAi to block Dip3, which increased DHYS and DOHH transcription (Figure $4 \mathrm{U}$ and $4 \mathrm{~V}$ ).

We speculated that due to the Dip3-mediated negative regulation of DHYS and DOHH via binding to promoter sites, vank proteins interacting with Dip3 exposed the promoter sites and triggered DHYS and $\mathrm{DOHH}$ transcription. Based on previous observations, we hypothesized that Dip3 interacted with the promoter DNA of DHYS and DOHH. Furthermore, an electrophoretic mobility shift assay (EMSA) helped us demonstrate how Dip3 and Vank86 proteins were expressed and purified (Figures 4W and 4X) by cloning promoter fragments (Figure S6B and S6C). EMSA showed that Dip3 was bound to the promoter of both genes. Besides, Vank-Dip3, but not Vank86, triggered DHYS and DOHH expression (Figures 4Y4Z2). In conclusion, these results showed that viral-elF5A-hypusination-dependent protein positively regulated DHYS and DOHH transcription. 
Bracovirus activates hypusination via elF5A nuclear-cytoplasmic transport

Next, we investigated how MbBV regulates hypusine modification. Hypusine modification of elF5A dictates its location in the cytoplasm, where it is required for protein synthesis (Lee et al., 2009). Ectopic expression of elF5A, DHYS, and DOHH did not change elF5A hypusination (Figure 5A), and hallmark proteins, P53, CypA, and CypD, were expressed following the same pattern, except for CypJ, which overexpressed DOHH (Figures 5A-5F; Figures S7A-S7C), meanings these protein level did not affect elF5A hypusination. We proposed the idea that hypusination may involve other mechanisms that are not specific for proteins. The next question is whether MbBV exercised its protein synthesis function by affecting hypusine localization. We performed western blotting to detect the localization of hypusine modification-related factors of elF5A in High Five cells under MbBV stimulation. We found that elF5A was primarily located in the nucleus and transported to the cytoplasm during MbBV infection (Figures 5G-5I), whereas DHYS (Figure 5J) was only located in the cytoplasm not nuclear. Further, immunofluorescence analysis showed that elF5A was predominantly localized in the nucleus, while during MbBV infection, elF5A is transported to the cytoplasm (Figures $5 \mathrm{~K}$ and $5 \mathrm{~L}$ ), whereas DHYS and DOHH remain in the cytoplasm (Figure 7SD). These results collectively demonstrated that MbBV functions by facilitating elF5A transport from the nucleus to the cytoplasm.

\section{Bracovirus integration-mediated hypusination drives persistence apoptosis}

Hypusination is required for specific protein expression and is accompanied by cell apoptosis, as evidenced by the expression of apoptosis-related proteins, P53, CypA, CypD, and CypJ. The next question is whether hypusination is required to maintain persistent apoptosis. We utilized parasitization to activate bracovirus integration-mediated hypusination $(\mathrm{BIMH})$ and an RNAi-mediated silencing approach to inhibit elF5A hypusination and detect apoptotic cells. In vivo, regardless of the proteins level, elF5A, P53, CypA, CypD and CypJ, of hemocytes of $S$. litura larvae did not differ between non-parasitism and BIMH, except for the hypusination, which showed a significant lower BIMH compared with non-parasitism (Figures 6A-6C and S8A-S8C), which suggests a continuous expression of apoptotic related proteins during BIMH. In vivo, inhibition of DHYS and DOHH decreased early apoptosis in both non-parasitism and BIMH (Figures 6D, 6E and 6F); however, inhibited elF5A decreased early apoptosis only in the BIMH condition (Figure $6 \mathrm{H}$ ). In later apoptosis, there were significant differences in non-parasitism and no differences were observed in the BIMH (Figures $6 \mathrm{~F}, 6 \mathrm{H}$, and $6 \mathrm{~J}$ ), suggesting that hypusination inhibition halted early apoptosis, a mechanism required to maintain persistent apoptosis.

We also examined the effect of the hypusination pathway on development. The head capsule development experiment showed that its development was significantly inhibited by parasitism, and the development of the head capsule was also significantly inhibited by elF5A silencing (Figure S9A). However, there was no significant difference between groups with silenced elF5A after parasitism and the 
control group (Figure S9B). Similar results were observed in the DHYS (Figures S9C and S9D) and DOHHsilenced conditions (Figures S9E and S9F). These data indicated that the silencing of the hypusine pathway affected the immunosuppression mediated by $M$. bicoloratus natural parasitism and caused no significant difference in head capsule width in S. litura.

These results collectively showed that integrated MbBV-mediated elF5A hypusination is required to drive cell apoptosis (Figure 6K).

Bracovirus fragments in $A B s$ re-integrate into recipient cells

Recently our study showed that MbBV-mediated innexin-hemichannel closure causes apoptotic cell disassembly; these results make a step forward from MbBV-induced apoptosis via elF5A hypusination. However, a few questions remain to be answered such as why did recipient cells present apoptosis and dismigration during immunosuppression (Figure S10)? And can bracovirus-mediated ABs transmit viral genes to recipient cells?

We speculated that viral gene fragments are transmitted between cells by ABs as carriers. We used PCR to detect viral gene fragments in the genome of Sf9 recipient cells incubated with MbBV-induced ABs and found that vank86, vank92, and ptp66 were present in recipient cell genome (Figure 7A). The existence of viral genes in the recipient cell genome indicates these genes may be transcribed. Therefore, we detected the transcription of viral genes, including vank86, vank92, vank101, and ptp66, in Sf9 recipient cells (Figures 7B and 7C). The transcription of viral genes, including vank86 and vank101, was detected in High Five recipient cells (Figure 7D). However, MbBV mediates dsDNA breaks (Luo and Pang, 2006b) that can damage the HIM; thus, how viral fragments without HIM integrate into the genome of recipient cells is currently unknown. The homologous recombination repair system mediates the integration of exogenous DNA (Brigulla and Wackernagel, 2010), especially when these exogenous DNA fragments carry homologous DNA sequences that can improve the integration efficiency (Figure 7E) (Simpson et al., 2007). Thus, we equally divided ABs into two parts. Half of the ABs were treated with RI-2 (inhibitor of RAD51), while the other half was not. Recipient cells incubated with UV-induced ABs were used as a negative control. After the ABs were incubated with recipient cells for $72 \mathrm{~h}$, the transcription levels of the viral genes were significantly decreased following RI-2 treatment in both Sf9 and High Five cells (Figures 7F and 7G). Compared with the negative control, the transcription level of the $\mathrm{RI}-2$ treatment group was significantly increased. These results suggested that apoptotic body-mediated viral gene transfer was related to the homologous recombination repair system but was not completely dependent on the homologous recombination repair system.

Next, we investigated the biological role of viral fragment reintegration. We have previously found that blocking the pl3K/Akt signaling pathway is essential for MbBV gene products to perform immunosuppressive function (Chen et al., 2021). To demonstrate that the virus genes are responsible for the physiological and biochemical changes in recipient cells, we examined the activation of the pl3K/Akt 
signaling pathway in both Sf9 and High Five recipient cells. The phosphorylation levels of pAkt-308 and pAkt-473 were significantly reduced in Sf9 recipient cells, and Cl-Caspase3 was significantly activated (Figures $7 \mathrm{H}$ and $7 \mathrm{l}$ ). Similarly, Akt phosphorylation was also detected in the High Five recipient cells and found that only pAkt-308 was dephosphorylated. In contrast, pAkt-473 presented high phosphorylation, and $\mathrm{Cl}$-Caspase-3 did not significantly differ in High Five recipient cells (Figure 7K). Considering that Akt requires pAkt-308 and pAkt-473 co-phosphorylation to perform signal transduction, only pAkt-308 dephosphorylation affected transduction, as evidenced by the inhibition of High Five cell migration.

In summary, viral gene products were transcribed and expressed after viral gene fragments re-integrate into recipient cells through ABs. Activated caspase-3 promoted Sf9 recipient cell apoptosis, and pAkt-308 dephosphorylation promoted High Five recipient cell dismigration. These observations indicate that gene products could still exert immunosuppression by regulating the PI3K/Akt signaling pathway (Figure 7L).

\section{Discussion}

Immunosuppressive signaling transduction is a novel aspect underlying immunity mechanisms. The PDV virus family usually cannot replicate after infecting host cells due to their special replication and symbiosis mechanism (Bezier et al., 2009; Burke and Strand, 2012; Burke et al., 2013; Gundersen-Rindal et al., 2013; Wetterwald et al., 2010). In addition, although PDV can cause persistent immunosuppression in lepidopterans, the specific mechanism remains unclear. This study explored the effect of MbBV on immunosuppressive signaling transmission. The results showed that MbBV-mediated ABs transmitted viral fragments to recipient cells, causing persistent immunosuppression. MbBV integration triggered elF5A hypusination to translate apoptosis-related and viral proteins; the former maintained persistent apoptosis, the latter positively activated elF5A hypusination pathway. MbBV induced dsDNA breaks in viral fragments delivered to recipient cell via $A B s$, which reintegrated to regulate pl3K-Akt phosphorylation to suppress immune responses, leading to recipient cell apoptosis and dismigration. To the best of our knowledge, this is the first report exploring the mechanism underlying immunosuppression transmission by MbBV-mediated ABs.

We provide evidence that MbBV-infected $S f 9$ cell-derived ABs can inhibit cell migration after phagocytosis by High Five recipient cells. Similarly, ABs can induce apoptosis after being engulfed by Sf 9 recipient cells. However, there was no change in the phenotype of virus infection inhibiting cell proliferation in this process, indicating that $A B s$ affect the physiological and biochemical functions of cells by means other than virus particle transmission.

Reportedly, MbBV integration triggers elF5A hypusination. Our findings demonstrate that MbBV DNA integration sets a foundation for viral gene transcription and plays an essential role in suppressing the host immune response at the DNA level. Moreover, MbBV DNAs separately integrate into different sites on different chromosomes, such as MdBV and CcBV (Beck et al., 2011; Chevignon et al., 2018). However, the connection between viral DNA and immunosuppression remains nebulous. Researchers believe that DNA integration of PDVs is the basis of the expression of viral genes that function as immune suppressors 
and development inhibitors (Burke and Strand, 2012; Strand and Burke, 2014). MbBV requires a structural foundation to complete its DNA integration, such as MdBV and CcBV (Chevignon et al., 2018). A comparative analysis of MbBV, MdBV, and CcBV genomes revealed that MbBV has HIMs on 15 of its 17 dsDNA circles. Although all HIMs are different, they have similar structures, containing two boundary sites on both the 5 terminals, homologous junction 1 and junction 2 near boundary sites, and a non-homologous loop in the middle (Chevignon et al., 2018). We found that HIMs of MbBV harbor all these structures, and HIMs on Circle 14, Circle 16, and Scaffold F157 have the highest integration quantity. This phenomenon corroborates the findings from our previous report on the integration and expression of Vank86 on Circle 14 and Vank101 on Circle 16 (Yu et al., 2016). Previous studies have shown that the whole DNA circle of PDV can be integrated into the host genome (Beck et al., 2011; Chevignon et al., 2018).

Hypusinated elF5A is an essential protein maintaining the viability of cells whose proposed function is to prevent the stalling of ribosomes during translation elongation (Martella et al., 2020). Previous studies have reported that proteins rich in proline, glycine, and charged amino acids are prone to ribosomal stagnation (Pelechano and Alepuz, 2017). The parasitism of M. bicoloratus inhibits the translation initiation factor elF4F (Cui et al., 2019; Dong et al., 2017), required for the usual translation of S. litura, but some host proteins and viral proteins are continuously expressed. Previous studies have reported that hypusinated elF5A specifically regulates the synthesis of the master autophagy and lysosome transcription factor, transcription factor EB (TFEB) (Zhang and Simon, 2020). However, whether hypusinated elF5A explicitly promotes the expression of viral and host proteins remained unclear. Viruses induce immune evasion through EV functions (Ramakrishnaiah et al., 2013), however, most previous studies have focused on mammalian viruses (Batagov and Kurochkin, 2013; Canitano et al., 2013; KeryerBibens et al., 2006; Pegtel et al., 2010; Ramakrishnaiah et al., 2013), hence data regarding insect viruses, especially the PDV family, remain limited. Our results prove that hypusine positively regulates the expression of proline-rich, glycine, and charged amino acid proteins.

When MbBV modifies hypusination, elF5A translocates from the nucleus to the cytoplasm to promote its activation in the cytoplasm. Previous studies have reported that the HIV-1 Rev transactivator protein mediates the translocation of viral mRNAs from the nucleus to the cytoplasm, which is essential for expressing viral structural proteins (Malim et al., 1990). elF5A specifically binds to Rev (Ruhl et al., 1993), and eIF5A loss-of-function mutants block the nuclear export of Rev protein and HIV1 replication (Bevec et al., 1996). We hypothesized that the MbBV-mediated translocation of elF5A from the nucleus to the cytoplasm promotes elF5A activation by enabling elF5A to carry viral proteins or host protein mRNA out of the nucleus, providing a large amount of substrate for protein translation.

The host elF5A hypusination cells mediated by MbBV provide apoptotic cells to generate ABs, which contain the virulence genes of MbBV, are used to perform immunosuppressive functions, and affect the physiological and biochemical activities of cells (Glatz et al., 2004; Summers and Dib-Hajj, 1995). In related studies, the expression of virulence genes can be detected continuously in bracovirus-infected cell lines (Bitra et al., 2016; Chevignon et al., 2018). Therefore, we hypothesized that unlike proteins and 
mRNAs with a short half-life, DNA could be easily transmitted between infected and uninfected cells (Baudrimont et al., 2017; Eden et al., 2011).

The key to the normal operation of the immune function lies in the activation of immune-related signaling pathways (Zhang et al., 2013). However, our previous studies have shown that MbBV performs an immunosuppressive function by inhibiting the pl3K/Akt signaling pathway(Chen et al., 2021). Here, we found that the phosphorylation sites p308 and p473 of pl3K/Akt in Sf9 recipient cells, co-incubated with ABs from infected Sf9 cells, were inhibited, thereby activating Cl-caspase3 activation. Meanwhile, the p308 phosphorylation site of pl3K/Akt in the High Five recipient cells was inhibited. Activation of the pl3K/Akt signaling pathway requires co-phosphorylation of two phosphorylation sites (Blanco et al., 2020). Therefore, the inhibition of either two or a single phosphorylation site can lead to immune inactivation.

Overall, our data provide a preliminary basis explaining how ABs participate in bracoviruses-mediated persistent immunosuppression. In addition, we found that MbBV can use ABs to escape immune surveillance. Whether other members of the PDV family can also transmit immunosuppressive signals between infected and uninfected cells through ABs warrants further studies.

\section{Declarations}

\section{ACKNOWLEDGMENTS}

This work was supported by the Science and Technology Planning Project in Key Areas of Yunnan Province [grant number 202001BB050002], National Natural Science Foundation of China [grant numbers $32160662,31772225,31471823,31260448$, and 31060251], NSFC-NRF [grant number 31411140238 to K.L.], and Yunnan Department of Science and Technology [grant number $2013 F A 003$ to K.L.]. K.L. was supported by the Donglu Scholar Program of Yunnan University.

\section{AUTHOR CONTRIBUTIONS}

K.L. conceived and supervised the study. G.Z. and X.Y. determined elF5A hyousination assays. C.C. and N.P. prepared apoptotic bodies (Abs) and perform ABs transmitting immunosuppressive assays. Q.C. and X.L. performed MbBV integration assays and genome sequencing and analysis. J.C., and Q.C., performed proteomics assays. Conceptualization, K.L.; Methodology, K.L., G.Z., C.C., Q.C., and J.C.; Investigation, G.Z., C.C., Q.C., X.Y., N.N., X.L., J.C., Y.H, Q.Z, J.M, C.C., H.T.; Writing-original draft, G.Z., C.C., Q.C., X.Y., N.N.; Writing-review \& editing, K.L., G.Z., X.Y., N.N.; Funding acquisition, K.L. All authors contributed to the manuscript preparation.

\section{DECLARATION OF INTERESTS}


The authors declare no competing interests.

\section{Methods Details}

\section{Reagents}

DHYS inhibitor GC7 was purchased from MedChemExpress (HY-108314A, MCE).

\section{Apoptosis analysis}

Analysis of apoptotic hemocytes was performed using an Annexin V-FITC/PI apoptosis detection kit (Vazyme, Nanjing, China) according to the manufacturer's instructions.

\section{Insect cultivation}

The S. litura colony was grown on an artificial diet at $27 \pm 1{ }^{\circ} \mathrm{C}$ and $60 \%-80 \%$ humidity (Li et al., 1998). The parasitoid M. bicoloratus colony was maintained on $S$. litura larvae grown in the laboratory. Adults were provided with honey as a dietary supplement (Luo et al., 2007).

\section{Cell lines and transfection}

The S. litura (Spli221) (Yanase et al., 1998), S. frugiperda (Sf9) (Vaughn et al., 1977), and cabbage looper Trichoplusia ni (High Five; provided by Sun Yat-Sen University) (Granados et al., 1994) cell lines were cultured at $27^{\circ} \mathrm{C}$. All cell lines were cultured in TNM-FH insect culture medium containing $10 \%$ fetal bovine serum (HyClone; Cytiva, Marlborough, MA, USA), as previously described (Liu et al., 2013). Cells were transfected using a 4:1 ratio of X-trem Fugene Transfection Reagent (4 mL; Roche, Basel, Switzerland) and $1 \mathrm{mg}$ DNA per well $(1 \mathrm{~mL})$ according to the manufacturer's protocol. Transfection efficiencies ranged from $50 \%$ to $75 \%$ in different cell lines, as measured by GFP expression.

\section{Induction, purification and isolation of apoptotic bodies}

Induction of apoptotic bodies: Sf9 cells were placed in each well of a 6-well plate. After $2 \mathrm{~h}$ of attachment, four equivalent MbBV virus and three generations of reBac-Inx3 at $5 \%$ concentration were added. The culture was then continued for $72 \mathrm{~h}$. In the control group, apoptosis was induced by UV irradiation, and the adherent cells were irradiated under a UV lamp (107 uw /) for $2 \mathrm{~h}$ and then cultured for $70 \mathrm{~h}$. The apoptotic body-induced cells were resuspended and placed into a centrifuge tube. They were centrifuged at $500 \times g$ for $5 \mathrm{~min}$ at room temperature. The supernatant was transferred to a new 
centrifuge tube and then centrifuged at $3000 \times g$ for $20 \mathrm{~min}$ at room temperature. The supernatant was discarded, and the pellet was resuspended with 1× phosphate-buffered saline (PBS) and centrifuged at $3000 \times g$ for $20 \mathrm{~min}$ at room temperature. The above steps were repeated one more time. The precipitates were collected, and the apoptotic bodies were resuspended in PBS and filtered using a $5-\mu \mathrm{m}$ filter. The filtrate was centrifuged at $3000 \times g$ for $20 \mathrm{~min}$ at room temperature, and the precipitate was collected as apoptotic bodies.

\section{Dilution of apoptotic bodies}

The apoptotic bodies obtained by the above method were gently resuspended and mixed with $1 \mathrm{~mL} 10 \%$ bovine fetal serum medium. Several centrifuge tubes were taken, and different volumes of $10 \%$ fetal bovine serum medium $(1.9,1.8,1.7,1.4$, and $1.2 \mathrm{~mL})$ were added. In addition, $10 \%$ bovine fetal serum medium containing apoptotic bodies was added into the corresponding centrifuge tubes $(0.1,0.2,0.3,0.6$, and $0.8 \mathrm{~mL}$ ) and gently mixed with a pipette gun for reserve use.

\section{Scratch test}

In total, High Five cells were placed in each well of a 6-well plate and allowed to grow to cover the bottom of the 6-well plate. Using a $100 \mu \mathrm{L}$ pipette, a cross line was marked at the bottom of the 6-well plate with the tip of a pipette. The apoptotic bodies collected after different apoptotic induction treatments were added to the 6-well plate and observed and photographed under a microscope. After $24 \mathrm{~h}$ of growth, they were observed again under a microscope and photographed. Image $\mathrm{J}$ was used to calculate the wound healing area within $24 \mathrm{~h}$.

\section{Characterization of apoptotic body infection induced by reBac-Inx3}

In a 6-well plate, Sf9 cells were placed in each well. Cells were infected with $5 \%$ reBac-Inx3 baculovirus of P3 generation for $72 \mathrm{~h}$. The above cells were blown up, and the apoptotic bodies were purified and collected by gradient centrifugation. The apoptotic bodies were resuspended with $1 \mathrm{~mL}$ of $10 \%$ bovine fetal serum medium and mixed thoroughly. The apoptotic bodies suspension $(500 \mu \mathrm{L})$ was extracted for protein quantification. The untreated SF9 cells were incubated with another $500 \mu \mathrm{L}$ of the apoptotic body solution for $72 \mathrm{~h}$. After incubation, the cells were washed twice with PBS and then collected for protein quantification.

\section{Hemocytes isolation}


Hemolymph $(500 \mu \mathrm{L})$ isolated from $\sim 100$ larvae of $S$. litura was centrifuged at $4^{\circ} \mathrm{C}$ for 5 min at $1000 \times g$, and pellets were collected as hemocytes. The pellets were centrifuged for $5 \mathrm{~min}$ at $1000 \times g$, washed with $1 \times$ PBS twice, and resuspended in $1 \mathrm{~mL}$ PBS. Hemocytes isolated from parasitized larvae were referred to as parasitized samples, and those from unparasitized larvae were used as controls (Zhang et al., 2019).

\section{Genome sequencing and analysis}

The samples were sequenced using an Illumina Hiseq 2000, and the total number of bases sequenced was greater than $3 \mathrm{Gbp}$. de novo DNA-seq assembly was performed using BWA, Velvet and ABySS software (Abuin et al., 2016; Simpson et al., 2009; Zerbino and Birney, 2008). The viral DNA sequences contained in the total DNA-seq were assembled by using SOAPdenovo (Xie et al., 2014), Platanus (Kajitani et al., 2014) and Cap3 (Huang and Madan, 1999). GeneMark was used to identify the functional proteins from the isolated contigs (Lukashin and Borodovsky, 1998). Genome sequences were used to blast with genome of MbBV (Yu et al., 2016) and genome of normal S. litura larvae (Cheng et al., 2017) by using Blast (v 2.6.0+) (Altschul et al., 1990).

\section{$\mathrm{MbBV}$ isolation and cell infection}

MbBV viral particles were purified as previously described (Luo and Pang, 2006b). Briefly, fresh wasps were frozen at $-20^{\circ} \mathrm{C}$ for $10 \mathrm{~min}$ and then placed on ice. The reproductive tracts of the female wasps were excised under a binocular stereomicroscope, and the separated ovaries were collected into a 1.5-mL Eppendorf tube on ice. The calyces were then punctured using forceps, the calyx fluid was resuspended in $1 \times$ PBS, and the resuspension was ground using a $2.5-\mathrm{mL}$ syringe. The mixture was centrifuged for $3 \mathrm{~min}$ at $1,000 \times g$ at $4{ }^{\circ} \mathrm{C}$ to remove the eggs and cellular debris. A $0.45-\mu \mathrm{m}$ syringe filter was used to purify the virions. Spli221 cells $\left(1.5 \times 10^{5}\right)$ were seeded in a 12-well culture plate (Corning Inc., Corning, NY, USA) $2 \mathrm{~h}$ before infection. The virions from one wasp could infect $1 \times 10^{5} \mathrm{Spli2} 21$ cells. Purified virions were added to each well in $1 \times 10^{5}$ cells/one wasp-derived MbBV equivalents, as previously described.

\section{Apoptosis analysis of hemocytes}

Briefly, after seven days of RNAi feeding, hemocytes $\left(1 \times 10^{5}\right)$ were extracted from S. litura larvae $(\mathrm{n}=3)$, resuspended in $100 \mu \mathrm{L}$ of $1 \times$ binding buffer, and incubated with $5 \mu \mathrm{L}$ of Annexin V-FITC dye and $5 \mu \mathrm{L}$ of PI dye for 10 min on ice in the dark. After adding $400 \mu \mathrm{L}$ of $1 \times$ binding buffer, an Olympus IX71 inverted fluorescence microscope with FV10-ASW 4.0 Viewer software (Olympus) was used to detect the apoptotic hemocytes. Early apoptotic cells identified by Annexin V-FITC showed green fluorescence, while late apoptotic cells stained by PI showed both green and red fluorescence. Five fields of more than 100 cells within each well of a 12-well plate were captured for analysis and quantification; experiments were 
performed in triplicate, and we obtained 15 images (from three wells) for each treatment for visual inspection and data quantification.

\section{Total DNA extraction}

Total DNA was isolated from hemocytes and Spli221 cells. Cells $\left(2 \times 10^{6}\right)$ were incubated in $200 \mu \mathrm{L}$ of lysis buffer (100 mM NaCl, $10 \mathrm{mM}$ Tris/ HCl, $25 \mathrm{mM}$ EDTA, $0.5 \%$ SDS, pH 8.0) containing $2.5 \mathrm{mg}$ of proteinase $\mathrm{K}$ per $\mathrm{mL}, 8 \mu \mathrm{L}$ of $20 \%$ Sarcosyl solution, and $1 \mathrm{mg}$ of RNaseA per $\mathrm{ml}$ at $55^{\circ} \mathrm{C}$ for $5 \mathrm{~h}$. The isolated DNA was further purified by phenol-chloroform extraction and subsequent ethanol precipitation. The concentration of each DNA sample was determined by measuring the optical density at A260/A280 using a NanoDrop 2000 and using $1 \times$ TBE agarose gels. DNA was prepared for sequencing and further PCR amplification. High quality samples (with an A260/A280 ratio $\geq 2.0, A_{260} / A_{230} \geq 2.0$, concentration $\geq 500 \mathrm{ng} / \mu \mathrm{L}$ ) were stored at $-20^{\circ} \mathrm{C}$ until use. DNA was prepared from at least three biological replicates.

\section{Proteomics of parasitised haemocytes, and MbBV infected Spli221 cells}

MbBV infected Spli221 cells were isolated, and protein sequences, for gene expression were determined using tandem mass spectrometry to examine the expression of genes of the parasitised haemocytes.

\section{Construction of elF5A, DHYS, and DOHH expression plasmids}

For elF5A, DHYS and DOHH overexpression, the corresponding genes were amplified by PCR using cDNA as a template and the following primers: E-eIF5A-F (5'-GAA TTC ATG GCT GAT ATC GAG GA-3') and EelF5A-R (5'-GCG GCC GCA TTT GTC AA-3'), containing EcoRl and Notl sites (underlined); E-DHYSF (5'-GGT ACC ATG GAT ATA ACT TCA GCT A-3') and E-dip3-R (5'-TCT AGA TAA ACA TTC TTT TTA TTG C-3'), containing $K p n l$ and $X$ bal sites (underlined), and E-DOHHF (5'-GAG CTC ATG GCA AAA GCT AG-3') and EDOHH-R (5'-TCT AGA CAG CCC TCG ACA GT-3'), containing SaCl and Xbal sites (underlined). The genes were directionally cloned into the pMD19 vector (Takara Bio, Kusatsu, Japan), and the inserts were confirmed by direct sequencing. Finally, the eukaryotic expression plasmid pIZT/V5-His (Invitrogen, Carlsbad, CA, USA) was used for the expression of elF5A, DHYA and DOHH fusion proteins with V5 and 6 His tags.

\section{Antibodies and Western blot}

Western blotting was performed as described (Liu et al., 2013). The following primary antibodies were used: mouse anti-V5 (1:5,000; Thermo Fisher Scientific, Waltham, MA, USA), mouse anti-tubulin (1:2,000; 
Solarbio, Beijing, China), rabbit anti-GAPDH (1:2,000; Solarbio), and rabbit anti-Hypusine (EMD Millipore; 1:1,000) antibodies, rabbit anti-elF5A (1:1,000; ABclonal), rabbit anti-DHPS (1:200; abcam), rabbit antiDOHH (1:500; Sigma-Aldrich). Secondary antibodies used were goat anti-mouse horseradish peroxidaseconjugated secondary antibody (1:2,000; Beyotime, Shanghai, China) and goat anti-rabbit horseradish peroxidase-conjugated secondary antibody (1:5,000). Anti-His (M1001020, Solarbio), Anti-pAKT473 (GB13012-3, Servicebio), Anti-pAKT308 (ab66134, Abcam), Anti-ATPaseß (GL Binchem synthesis), AntiInx1(GL Binchem synthesis), Anti-Inx2 (GL Binchem synthesis), Anti-Inx3(GL Binchem synthesis), AntiInx4 (GL Binchem synthesis), Cleaved-Caspase (WL02117, Wanleibio), Anti-GAPDH (M1000110, Solarbio), Anti- $\beta$ Tubulin (AF1216, Beyotime), Goat Anti-Mouse (A0216, Beyotime), Goat Anti-Rabbit (A0208, Beyotime). Proteins were semi-quantified via densitometry using ImageJ (National Institutes of Health, Bethesda, MD, USA).

\section{Electrophoretic Mobility Shift Assay (EMSA) and Nonlinear regression curve and Kd value determination}

The promoter prediction, the eukaryotic protein expression, and EMSA were performed as described (Bhaskar and Courey, 2002; Crowe et al., 1994; Hellman and Fried, 2007). First, we used the website http://gene-regulation.com to analyze the domain binding of the promoter region sequences of DOHH and DHYS. We screened out the gene sequences that may interact with the myb/SANT domain of Dip3. Then, through the designed primers, the target gene was obtained by PCR from the whole genome of S. litura hemolymph. Subsequently, we sonicated the BL21(DE3) Escherichia coli carrying pET28a HisDip3 and pET28a His-Vank86 prokaryotic expression vector, and extracted the crude protein samples of His-Dip3 and His-Vank86. We then immediately purified the crude protein samples obtained above by nickel magnetic beads to obtain purified His-Dip3 and His-Vank86 samples. Finally, we took the purified His-Dip3 and His-Vank86 proteins, set four gradients $(0,5,10,20 \mu \mathrm{g})$, added $1 \mu \mathrm{g}$ of target DNA and $10 \times$ Binding Buffer, and incubated the mixture at room temperature for $30 \mathrm{~min}$. The samples were run on (100 V $30 \mathrm{~min}$ ) agarose gel, to observe the results..

Nonlinear regression curve and $\mathrm{K}_{\mathrm{d}}$ value determination were analyzed as described (Schneider et al., 2012; Seo et al., 2019). We used the ImageJ program to analyze the agarose gel image by inverting the image first, and then use ImageJ to measure the raw integrated density (RID) of each band. The results were exported to an Excel table to perform the following calculations. Each RID value was subtracted from the lowest RID value, and the lowest RID value was subtracted from itself, and the result was zero. These values are called relative RIDs. Each relative RID value was divided by the largest relative RID and then multiplied by 100 . The maximum relative RID value was divided by itself, multiplied by 100 , and the result was 100 . These values are called percentage RID values. Finally, the percentage RID value was subtracted from 100 to get the binding rate. The obtained binding rate was analyzed using GraphPad Prism. An XY list was created, the protein concentration being in the $\mathrm{X}$ list, and the binding rate being in the $Y$ list. The analysis option was selected, the equation labeled "a site-specific binding" was chosen, and the $K_{d}$ value was calculated to get a nonlinear regression line.

Page $18 / 36$ 


\section{Immunofluorescence}

Immunofluorescence was performed as previously described (Liu et al., 2013), with minor modifications. High Five Cells grown on coverslips were fixed in $4 \%$ paraformaldehyde for $15 \mathrm{~min}$ and permeabilized in $0.2 \%$ Triton X-100 in PBS (PBST). The fixed cells were blocked with $5 \%$ normal goat serum in $0.1 \%$ PBST for $15 \mathrm{~min}$. Ectopically expressed V5-fused elF5A were identified using a mouse anti-V5 antibody (1:2,000; Thermo Fisher Scientific) and AlexaFluor ${ }^{\circledR} 568$ Goat anti-mouse IgG $(H+L)(1: 2,000$; Thermo Fisher Scientific). Labeled cells were incubated with phalloidin (Sigma-Aldrich, St. Louis, MO, USA) diluted 1:40 in PBS for $1 \mathrm{~h}$ at $37^{\circ} \mathrm{C}$. Cells were then washed with PBS and incubated with 4, 6-diamidino-2phenylindole (DAPI; 1:1,000; Roche) for 5 min. Slides were mounted with mounting medium, antifading (Cat. No. S2100; Solarbio). Cells were imaged using Confocal (ZEISS LSM 800) microscopy.

\section{Plasmid construction for dsRNA feeding}

Plasmids were constructed as described previously (Timmons et al., 2001). The sequences encoding the elF5A, DHYS and DOHH genes were inserted into the RNAi vector L4440, containing two convergent T7 polymerase promoters in opposite orientation separated by a multiple cloning site.

\section{Preparation of dsRNA and dsRNA feeding}

The plasmids for dsRNA feeding were transformed into the bacterial host E. coli HT115 (DE3). In brief, a single colony of HT115 containing the recombinant L4440 vector was inoculated in $4 \mathrm{~mL}$ of LB medium containing $4 \mu \mathrm{L}$ ampicillin $(100 \mu \mathrm{g} / \mathrm{mL})$ and $4 \mu \mathrm{L}$ tetracycline $(100 \mu \mathrm{g} / \mathrm{mL})$ and cultured overnight at $37^{\circ} \mathrm{C}$. The cultures were diluted to reach an $\mathrm{OD}_{600}$ of 0.4 . Isopropyl- $\beta$-D-thiogalactopyranoside (IPTG) was then added at a final concentration of $0.8 \mathrm{mM}$, and the cultures were incubated for $4 \mathrm{~h}$ with shaking at 37 ${ }^{\circ} \mathrm{C}$. An aliquot of the suspension $(200 \mathrm{~mL})$ was centrifuged at $10,000 \times \mathrm{g}$ for $10 \mathrm{~min}$; the pellet $\left(\mathrm{OD}_{600}\right.$ $\approx 1$ ) was resuspended in $5 \mathrm{~mL}$ of sterile $\mathrm{H}_{2} \mathrm{O}$ after cooling to $50^{\circ} \mathrm{C}$ and mixed with $50 \mathrm{~mL}$ of freshly prepared artificial feed. The prepared dsRNA feed was stored in a small plastic box $(9.5 \times 7.0 \times 5.5 \mathrm{~cm}$, $200 \mathrm{~mL}$ ) at $4{ }^{\circ} \mathrm{C}$ and used within one week. Control $S$. litura received a standard feed. Before hatching, $S$. litura eggs were placed on artificial food. For long-term maintenance of the dsRNA diet, larvae were provided with fresh food every day (Dong et al., 2017). Hemocytes were harvested on day 7 for apoptosis assays. Head capsule measurement was performed daily. EGFP dsRNA was used as control dsRNA.

\section{RT-qPCR}


The recipient cells incubated with apoptotic bodies were collected. Total RNA was isolated from the samples using the RNAiso Plus kit (Takara Bio) and then treated with DNA enzymes. NanoDrop 2000 was used to measure the concentration and $\mathrm{OD}_{260} / \mathrm{OD}_{280}$ of each RNA sample. CDNA was then synthesized by PrimeScript II 1st Strand cDNA Synthesis Kit (Takara Bio). All cDNA samples were stored at $-80^{\circ} \mathrm{C}$. The above-mentioned synthesized cDNA was used as a template for RT-qPCR analysis. The primers used

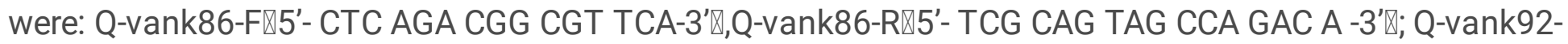

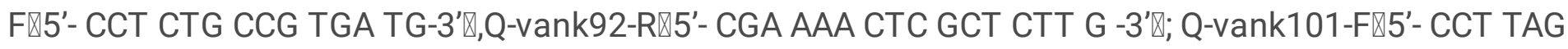

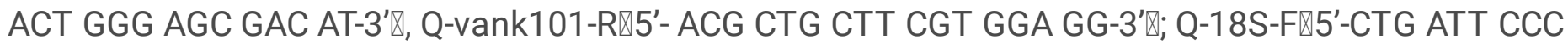

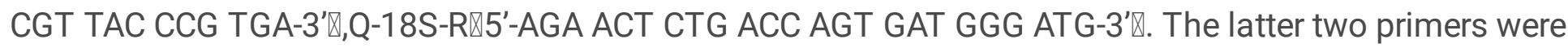
used to amplify $18 \mathrm{~S}$ as endogenous controls. Cycle parameters are as follows: $95^{\circ} \mathrm{C}$ for $30 \mathrm{~s} ; 40$ cycles of $95^{\circ} \mathrm{C}$ for $5 \mathrm{~s} ; 40$ cycles of $60^{\circ} \mathrm{C}$ for $34 \mathrm{~s} ; 95^{\circ} \mathrm{C}$ for $15 \mathrm{~s} ; 60^{\circ} \mathrm{C}$ for $1 \mathrm{~min} ; 95^{\circ} \mathrm{C}$ for $15 \mathrm{~s}$ and an indefinite hold at $10^{\circ} \mathrm{C}$. Each sample was repeated for 3 times. The relative gene expression was calculated by method.

\section{QUANTIFICATION AND STATISTICAL ANALYSIS}

Data were analyzed using GraphPad Prism (ver. 7, Prism), and statistical significance was determined using the Student's $t$-test for unpaired experiments (two-tailed). $p<0.05$ indicates statistically significant difference between groups. The resulting data are presented as means \pm SEM from at least three independent experiments.

\section{References}

Abbruzzese, A., Park, M.H., and Folk, J.E. (1986). Deoxyhypusine hydroxylase from rat testis. Partial purification and characterization. J Biol Chem 261, 3085-3089.

Abuin, J.M., Pichel, J.C., Pena, T.F., and Amigo, J. (2016). SparkBWA: Speeding Up the Alignment of HighThroughput DNA Sequencing Data. PLoS One 11, e0155461.

Akers, J.C., Gonda, D., Kim, R., Carter, B.S., and Chen, C.C. (2013). Biogenesis of extracellular vesicles (EV): exosomes, microvesicles, retrovirus-like vesicles, and apoptotic bodies. J Neurooncol 113, 1-11.

Altschul, S.F., Gish, W., Miller, W., Myers, E.W., and Lipman, D.J. (1990). Basic local alignment search tool. J Mol Biol 215, 403-410.

Artieri, C.G., and Fraser, H.B. (2014). Accounting for biases in riboprofiling data indicates a major role for proline in stalling translation. Genome Res 24, 2011-2021.

Batagov, A.O., and Kurochkin, I.V. (2013). Exosomes secreted by human cells transport largely mRNA fragments that are enriched in the 3'-untranslated regions. Biol Direct $8,12$. 
Baudrimont, A., Voegeli, S., Viloria, E.C., Stritt, F., Lenon, M., Wada, T., Jaquet, V., and Becskei, A. (2017). Multiplexed gene control reveals rapid mRNA turnover. Sci Adv 3, e1700006.

Beck, M., and Strand, M.R. (2005). Glc1.8 from Microplitis demolitor bracovirus induces a loss of adhesion and phagocytosis in insect high five and S2 cells. J Virol 79, 1861-1870.

Beck, M.H., Zhang, S., Bitra, K., Burke, G.R., and Strand, M.R. (2011). The encapsidated genome of Microplitis demolitor bracovirus integrates into the host Pseudoplusia includens. J. Virol. 85, 1168511696.

Bevec, D., Jaksche, H., Oft, M., Wöhl, T., Himmelspach, M., Pacher, A., Schebesta, M., Koettnitz, K., Dobrovnik, M., Csonga, R., et al. (1996). Inhibition of HIV-1 replication in lymphocytes by mutants of the Rev cofactor elF-5A. Science 271, 1858-1860.

Bezier, A., Annaheim, M., Herbiniere, J., Wetterwald, C., Gyapay, G., Bernard-Samain, S., Wincker, P., Roditi, I., Heller, M., Belghazi, M., et al. (2009). Polydnaviruses of braconid wasps derive from an ancestral nudivirus. Science 323, 926-930.

Bhaskar, V., and Courey, A.J. (2002). The MADF-BESS domain factor Dip3 potentiates synergistic activation by Dorsal and Twist. Gene 299, 173-184.

Bitra, K., Burke, G.R., and Strand, M.R. (2016). Permissiveness of lepidopteran hosts is linked to differential expression of bracovirus genes. Virology 492, 259-272.

Blanco, J., Cameirao, C., Lopez, M.C., and Munoz-Barroso, I. (2020). Phosphatidylinositol-3-kinase-Akt pathway in negative-stranded RNA virus infection: a minireview. Arch Virol 165, 2165-2176.

Brigulla, M., and Wackernagel, W. (2010). Molecular aspects of gene transfer and foreign DNA acquisition in prokaryotes with regard to safety issues. Appl Microbiol Biotechnol 86, 1027-1041.

Burke, G.R., and Strand, M.R. (2012). Deep sequencing identifies viral and wasp genes with potential roles in replication of Microplitis demolitor Bracovirus. J Virol 86, 3293-3306.

Burke, G.R., Thomas, S.A., Eum, J.H., and Strand, M.R. (2013). Mutualistic polydnaviruses share essential replication gene functions with pathogenic ancestors. PLoS Pathog 9, e1003348.

Cai, Q.C., Chen, C.X., Liu, H.Y., Zhang, W., Han, Y.F., Zhang, Q., Zhou, G.F., Xu, S., Liu, T., Xiao, W., et al. (2021). Interactions of Vank proteins from Microplitis bicoloratus bracovirus with host Dip3 suppress elF4E expression. Dev Comp Immunol 118, 103994.

Canitano, A., Venturi, G., Borghi, M., Ammendolia, M.G., and Fais, S. (2013). Exosomes released in vitro from Epstein-Barr virus (EBV)-infected cells contain EBV-encoded latent phase mRNAs. Cancer Lett 337, 193-199. 
Chatzinikolaou, G., Karakasilioti, I., and Garinis, G.A. (2014). DNA damage and innate immunity: links and trade-offs. Trends Immunol 35, 429-435.

Chen, C.-X., He, H.-J., Cai, Q.-C., Zhang, W., Kou, T.-C., Zhang, X.-W., You, S., Chen, Y.-B., Liu, T., Xiao, W., et al. (2021). Bracovirus-mediated innexin-hemichannel closure in cell disassembly. iScience 24, 102281.

Chen, X.Y., Liu, J., Zhang, C.D., Li, Y.F., Liu, T.H., Wang, L., Yu, Q.Y., Zhang, Y.H., Lu, C., and Pan, M.H. (2015). The silkworm GSTe4 is sensitive to phoxim and protects HEK293 cells against UV-induced cell apoptosis. Bull Entomol Res 105, 399-407.

Chen, Y.-B., Xiao, W., Li, M., Zhang, Y., Yang, Y., Hu, J.-S., and Luo, K.-J. (2016). N-terminally elongated Splilnx2 and Splilnx3 reduce baculovirus-triggered apoptosis via hemichannel closure. Arch. Insect Biochem. Physiol. 92, 24-37.

Cheng, T., Wu, J., Wu, Y., Chilukuri, R.V., Huang, L., Yamamoto, K., Feng, L., Li, W., Chen, Z., Guo, H., et al. (2017). Genomic adaptation to polyphagy and insecticides in a major East Asian noctuid pest. Nat. Eco. Evol.

Chevignon, G., Periquet, G., Gyapay, G., Vega-Czarny, N., Musset, K., Drezen, J.-M., and Huguet, E. (2018). Cotesia congregata Bracovirus circles encoding PTP and Ankyrin genes integrate into the DNA of parasitized Manduca sexta hemocytes. J. Virol. 92, e00438-00418.

Crowe, J., Döbeli, H., Gentz, R., Hochuli, E., Stüber, D., and Henco, K. (1994). 6xHis-Ni-NTA chromatography as a superior technique in recombinant protein expression/purification. Methods Mol Biol 31, 371-387.

Cui, J.H., Dong, S.M., Chen, C.X., Xiao, W., Cai, Q.C., Zhang, L.D., He, H.J., Zhang, W., Zhang, X.W., Liu, T., et al. (2019). Microplitis bicoloratus bracovirus modulates innate immune suppression through the elF4EelF4A axis in the insect Spodoptera litura. Dev Comp Immunol 95, 101-107.

Dong, S.M., Cui, J.H., Zhang, W., Zhang, X.W., Kou, T.C., Cai, Q.C., Xu, S., You, S., Yu, D.S., Ding, L., et al. (2017). Inhibition of translation initiation factor elF4A is required for apoptosis mediated by Microplitis bicoloratus bracovirus. Arch Insect Biochem Physiol 96.

Dukers, D.F., Meij, P., Vervoort, M.B., Vos, W., Scheper, R.J., Meijer, C.J., Bloemena, E., and Middeldorp, J.M. (2000). Direct immunosuppressive effects of EBV-encoded latent membrane protein 1. J Immunol 165, 663-670.

Eden, E., Geva-Zatorsky, N., Issaeva, I., Cohen, A., Dekel, E., Danon, T., Cohen, L., Mayo, A., and Alon, U. (2011). Proteome half-life dynamics in living human cells. Science 331, 764-768.

Elfgang, C., Rosorius, O., Hofer, L., Jaksche, H., Hauber, J., and Bevec, D. (1999). Evidence for specific nucleocytoplasmic transport pathways used by leucine-rich nuclear export signals. Proc Natl Acad Sci U S A 96, 6229-6234. 
Eum, J.H., Bottjen, R.C., Pruijssers, A.J., Clark, K.D., and Strand, M.R. (2010). Characterization and kinetic analysis of protein tyrosine phosphatase-H2 from Microplitis demolitor bracovirus. Insect Biochem Mol Biol 40,690-698.

Flanagan, J., Middeldorp, J., and Sculley, T. (2003). Localization of the Epstein-Barr virus protein LMP 1 to exosomes. J Gen Virol 84, 1871-1879.

Gamble, C.E., Brule, C.E., Dean, K.M., Fields, S., and Grayhack, E.J. (2016). Adjacent Codons Act in Concert to Modulate Translation Efficiency in Yeast. Cell 166, 679-690.

Gardin, J., Yeasmin, R., Yurovsky, A., Cai, Y., Skiena, S., and Futcher, B. (2014). Measurement of average decoding rates of the 61 sense codons in vivo. Elife 3 .

Gitau, C.W., Gundersen-Rindal, D., Pedroni, M., Mbugi, P.J., and Dupas, S. (2007). Differential expression of the CrV1 haemocyte inactivation-associated polydnavirus gene in the African maize stem borer Busseola fusca (Fuller) parasitized by two biotypes of the endoparasitoid Cotesia sesamiae (Cameron). J Insect Physiol 53, 676-684.

Glatz, R.V., Asgari, S., and Schmidt, O. (2004). Evolution of polydnaviruses as insect immune suppressors. Trends Microbiol 12, 545-554.

Granados, R.R., Li, G., Derksen, A.C.G., and McKenna, K.A. (1994). A new insect cell line from Trichoplusia ni (BTI-Tn-5B1-4) susceptible to Trichoplusia ni single enveloped nuclear polyhedrosis virus \%J Academic Press. 64.

Gundersen-Rindal, D., Dupuy, C., Huguet, E., and Drezen, J.-M. (2013). Parasitoid polydnaviruses: evolution, pathology and applications. Biocontrol Science and Technology 23, 1-61.

Guo, L.E., Zhang, J.F., Liu, X.Y., Zhang, L.M., Zhang, H.L., Chen, J.H., Xie, X.G., Zhou, Y., Luo, K.-J., and Yoon, J. (2015). Phosphate ion targeted colorimetric and fluorescent probe and its use to monitor endogeneous phosphate ion in a hemichannel-closed cell. Anal. Chem. 87, 1196-1201.

Hellman, L.M., and Fried, M.G. (2007). Electrophoretic mobility shift assay (EMSA) for detecting proteinnucleic acid interactions. Nat Protoc 2, 1849-1861.

Holmgren, L., Bergsmedh, A., and Spetz, A.L. (2002). Horizontal transfer of DNA by the uptake of apoptotic bodies. Vox Sang 83 Suppl 1, 305-306.

Hoque, M., Hanauske-Abel, H.M., Palumbo, P., Saxena, D., D'Alliessi Gandolfi, D., Park, M.H., Pe'ery, T., and Mathews, M.B. (2009). Inhibition of HIV-1 gene expression by Ciclopirox and Deferiprone, drugs that prevent hypusination of eukaryotic initiation factor 5A. Retrovirology 6, 90.

Huang, X., and Madan, A. (1999). CAP3: A DNA sequence assembly program. Genome Res 9, 868-877. 
Ishfaq, M., Maeta, K., Maeda, S., Natsume, T., Ito, A., and Yoshida, M. (2012). The role of acetylation in the subcellular localization of an oncogenic isoform of translation factor elF5A. Biosci Biotechnol Biochem 76, 2165-2167.

Joe, Y.A., Wolff, E.C., and Park, M.H. (1995). Cloning and expression of human deoxyhypusine synthase cDNA. Structure-function studies with the recombinant enzyme and mutant proteins. J Biol Chem 270, 22386-22392.

Kajitani, R., Toshimoto, K., Noguchi, H., Toyoda, A., Ogura, Y., Okuno, M., Yabana, M., Harada, M., Nagayasu, E., Maruyama, H., et al. (2014). Efficient de novo assembly of highly heterozygous genomes from whole-genome shotgun short reads. Genome Res 24, 1384-1395.

Kalamvoki, M., Du, T., and Roizman, B. (2014). Cells infected with herpes simplex virus 1 export to uninfected cells exosomes containing STING, viral mRNAs, and microRNAs. Proc Natl Acad Sci U S A 111, E4991-4996.

Kerr, J.F., Wyllie, A.H., and Currie, A.R. (1972). Apoptosis: a basic biological phenomenon with wideranging implications in tissue kinetics. Br J Cancer 26, 239-257.

Keryer-Bibens, C., Pioche-Durieu, C., Villemant, C., Souquere, S., Nishi, N., Hirashima, M., Middeldorp, J., and Busson, P. (2006). Exosomes released by EBV-infected nasopharyngeal carcinoma cells convey the viral latent membrane protein 1 and the immunomodulatory protein galectin 9. BMC Cancer 6, 283 .

Kumar, S., and Kim, Y. (2016). Glyceraldehyde-3-phosphate dehydrogenase is a mediator of hemocytespreading behavior and molecular target of immunosuppressive factor CrV1. Dev Comp Immunol 54, 97108.

Labropoulou, V., Douris, V., Stefanou, D., Magrioti, C., Swevers, L., and latrou, K. (2008). Endoparasitoid wasp bracovirus-mediated inhibition of hemolin function and lepidopteran host immunosuppression. Cell Microbiol 10, 2118-2128.

Lee, S.B., Park, J.H., Kaevel, J., Sramkova, M., Weigert, R., and Park, M.H. (2009). The effect of hypusine modification on the intracellular localization of elF5A. Biochem Biophys Res Commun 383, 497-502.

Li, G.-H., Chen, Q.-J., and Pang, Y. (1998). Studies of artificial diets for the beet armyworm, Spodoptera exigua. Acta Scientiae Circumstan- tiae/Huanjing Kexue Xuebao 4, 1-5.

Li, M., Pang, Z., Xiao, W., Liu, X., Zhang, Y., Yu, D., Yang, M., Yang, Y., Hu, J., and Luo, K. (2014). A transcriptome analysis suggests apoptosis-related signaling pathways in hemocytes of Spodoptera litura after parasitization by Microplitis bicoloratus. PLoS One 9, e110967.

Lipowsky, G., Bischoff, F.R., Schwarzmaier, P., Kraft, R., Kostka, S., Hartmann, E., Kutay, U., and Görlich, D. (2000). Exportin 4: a mediator of a novel nuclear export pathway in higher eukaryotes. Embo j 19, 43624371. 
Liu, T., Li, M., Zhang, Y., Pang, Z., Xiao, W., Yang, Y., and Luo, K. (2013). A role for Innexin2 and Innexin3 proteins from Spodoptera litura in apoptosis. PLoS One 8, e70456.

Lukashin, A.V., and Borodovsky, M. (1998). GeneMark.hmm: new solutions for gene finding. Nucleic Acids Res 26, 1107-1115.

Luo, and Pang (2006a). Disruption effect of Microplitis bicoloratus polydnavirus EGF-like protein, MbCRP, on actin cytoskeleton in lepidopteran insect hemocytes. Acta Biochim Biophys Sin (Shanghai) 38, 577585 .

Luo, and Pang (2006b). Spodoptera litura multicapsid nucleopolyhedrovirus inhibits Microplitis bicoloratus polydnavirus-induced host granulocytes apoptosis. J Insect Physiol 52, 795-806.

Luo, K., Trumble, J.T., and Pang, Y. (2007). Development of Microplitis bicoloratus on Spodoptera litura and implications for biological control \%J BioControl. 52.

Malim, M.H., Tiley, L.S., McCarn, D.F., Rusche, J.R., Hauber, J., and Cullen, B.R. (1990). HIV-1 structural gene expression requires binding of the Rev trans-activator to its RNA target sequence. Cell $60,675-683$.

Malyutin, A.G., Musalgaonkar, S., Patchett, S., Frank, J., and Johnson, A.W. (2017). Nmd3 is a structural mimic of elF5A, and activates the cpGTPase Lsg1 during $60 \mathrm{~S}$ ribosome biogenesis. EMBO J 36, 854-868.

Martella, M., Catalanotto, C., Talora, C., La Teana, A., Londei, P., and Benelli, D. (2020). Inhibition of Eukaryotic Translation Initiation Factor 5A (elF5A) Hypusination Suppress p53 Translation and Alters the Association of elF5A to the Ribosomes. Int J Mol Sci 21.

Mathieu, M., Martin-Jaular, L., Lavieu, G., and Thery, C. (2019). Specificities of secretion and uptake of exosomes and other extracellular vesicles for cell-to-cell communication. Nat Cell Biol 21, 9-17.

Meckes, D.G., Jr., Gunawardena, H.P., Dekroon, R.M., Heaton, P.R., Edwards, R.H., Ozgur, S., Griffith, J.D., Damania, B., and Raab-Traub, N. (2013). Modulation of B-cell exosome proteins by gamma herpesvirus infection. Proc Natl Acad Sci U S A 110, E2925-2933.

Mohan, M. (2003). Antioxidants prevent UV-induced apoptosis by inhibiting mitochondrial cytochrome c release and caspase activation in Spodoptera frugiperda (Sf9) cells. Cell Biology International 27, 483490.

Olsen, M.E., and Connor, J.H. (2017). Hypusination of elF5A as a Target for Antiviral Therapy. DNA Cell Biol. 36, 198-201.

Olsen, M.E., Cressey, T.N., Muhlberger, E., and Connor, J.H. (2018). Differential Mechanisms for the Involvement of Polyamines and Hypusinated elF5A in Ebola Virus Gene Expression. J Virol. 
Park, J.H., Aravind, L., Wolff, E.C., Kaevel, J., Kim, Y.S., and Park, M.H. (2006). Molecular cloning, expression, and structural prediction of deoxyhypusine hydroxylase: a HEAT-repeat-containing metalloenzyme. Proc Natl Acad Sci U S A 103, 51-56.

Park, M.H. (2006). The post-translational synthesis of a polyamine-derived amino acid, hypusine, in the eukaryotic translation initiation factor 5A (elF5A). J. Biochem. 139, 161-169.

Park, M.H., Cooper, H.L., and Folk, J.E. (1981). Identification of hypusine, an unusual amino acid, in a protein from human lymphocytes and of spermidine as its biosynthetic precursor. Proc Natl Acad Sci U S A $78,2869-2873$.

Park, M.H., Nishimura, K., Zanelli, C.F., and Valentini, S.R. (2010). Functional significance of elF5A and its hypusine modification in eukaryotes. Amino Acids 38, 491-500.

Park, M.H., and Wolff, E.C. (2018). Hypusine, a polyamine-derived amino acid critical for eukaryotic translation. J Biol Chem 293, 18710-18718.

Pavlov, M.Y., Watts, R.E., Tan, Z., Cornish, V.W., Ehrenberg, M., and Forster, A.C. (2009). Slow peptide bond formation by proline and other N-alkylamino acids in translation. Proc Natl Acad Sci U S A 106, 50-54.

Pegtel, D.M., Cosmopoulos, K., Thorley-Lawson, D.A., van Eijndhoven, M.A., Hopmans, E.S., Lindenberg, J.L., de Gruijl, T.D., Wurdinger, T., and Middeldorp, J.M. (2010). Functional delivery of viral miRNAs via exosomes. Proc Natl Acad Sci U S A 107, 6328-6333.

Pelechano, V., and Alepuz, P. (2017). elF5A facilitates translation termination globally and promotes the elongation of many non polyproline-specific tripeptide sequences. Nucleic Acids Res 45, 7326-7338.

Pruijssers, A.J., and Strand, M.R. (2007). PTP-H2 and PTP-H3 from Microplitis demolitor Bracovirus localize to focal adhesions and are antiphagocytic in insect immune cells. J Virol 81, 1209-1219.

Ramakrishnaiah, V., Thumann, C., Fofana, I., Habersetzer, F., Pan, Q., de Ruiter, P.E., Willemsen, R., Demmers, J.A., Stalin Raj, V., Jenster, G., et al. (2013). Exosome-mediated transmission of hepatitis C virus between human hepatoma Huh7.5 cells. Proc Natl Acad Sci U S A 110, 13109-13113.

Ratajczak, J., Miekus, K., Kucia, M., Zhang, J., Reca, R., Dvorak, P., and Ratajczak, M.Z. (2006). Embryonic stem cell-derived microvesicles reprogram hematopoietic progenitors: evidence for horizontal transfer of mRNA and protein delivery. Leukemia 20, 847-856.

Ruhl, M., Himmelspach, M., Bahr, G.M., Hammerschmid, F., Jaksche, H., Wolff, B., Aschauer, H., Farrington, G.K., Probst, H., Bevec, D., et al. (1993). Eukaryotic initiation factor 5A is a cellular target of the human immunodeficiency virus type 1 Rev activation domain mediating trans-activation. J Cell Biol 123, 13091320. 
Sabi, R., and Tuller, T. (2015). A comparative genomics study on the effect of individual amino acids on ribosome stalling. BMC Genomics 16 Supp/ 10, S5.

Schneider, C.A., Rasband, W.S., and Eliceiri, K.W. (2012). NIH Image to ImageJ: 25 years of image analysis. Nature Methods: Techniques for life scientists and chemists 9.

Schuller, A.P., Wu, C.C., Dever, T.E., Buskirk, A.R., and Green, R. (2017). elF5A Functions Globally in Translation Elongation and Termination. Mol Cell 66, 194-205 e195.

Seo, M., Lei, L., and Egli, M. (2019). Label-Free Electrophoretic Mobility Shift Assay (EMSA) for Measuring Dissociation Constants of Protein-RNA Complexes. Current Protocols in Nucleic Acid Chemistry 76.

Shin, B.S., Katoh, T., Gutierrez, E., Kim, J.R., Suga, H., and Dever, T.E. (2017). Amino acid substrates impose polyamine, elF5A, or hypusine requirement for peptide synthesis. Nucleic Acids Res 45, 8392-8402.

Simpson, D.J., Fry, J.C., Rogers, H.J., and Day, M.J. (2007). Transformation of Acinetobacter baylyi in nonsterile soil using recombinant plant nuclear DNA. Environ Biosafety Res 6, 101-112.

Simpson, J.T., Wong, K., Jackman, S.D., Schein, J.E., Jones, S.J., and Birol, I. (2009). ABySS: a parallel assembler for short read sequence data. Genome Res 19, 1117-1123.

Soldan, K., Barbara, J.A., and Dow, B.C. (2002). Transfusion-transmitted hepatitis B virus infection in the UK: a small and moving target. Vox Sang 83, 305-308.

Stanley, D., Miller, J., and Tunaz, H. (2009). Eicosanoid actions in insect immunity. J Innate Immun 1, 282290.

Strand, M.R., and Burke, G.R. (2014). Polydnaviruses: Nature's Genetic Engineers. Annu Rev Virol 1, 333354.

Suhasini, M., and Reddy, T.R. (2009). Cellular proteins and HIV-1 Rev function. Curr HIV Res 7, 91-100.

Summers, M.D., and Dib-Hajj, S.D. (1995). Polydnavirus-facilitated endoparasite protection against host immune defenses. Proc Natl Acad Sci U S A 92, 29-36.

Taylor, D.D., and Gercel-Taylor, C. (2008). MicroRNA signatures of tumor-derived exosomes as diagnostic biomarkers of ovarian cancer. Gynecol Oncol 110,13-21.

Thoetkiattikul, H., Beck, M.H., and Strand, M.R. (2005). Inhibitor kappaB-like proteins from a polydnavirus inhibit NF-kappaB activation and suppress the insect immune response. Proc Natl Acad Sci U S A 102, 11426-11431.

Timmons, L., Court, D.L., and Fire, A. (2001). Ingestion of bacterially expressed dsRNAs can produce specific and potent genetic interference in Caenorhabditis elegans. Gene 263, 103-112. 
Valadi, H., Ekstrom, K., Bossios, A., Sjostrand, M., Lee, J.J., and Lotvall, J.O. (2007). Exosome-mediated transfer of mRNAs and microRNAs is a novel mechanism of genetic exchange between cells. Nat Cell Biol 9, 654-659.

Vaughn, J.L., Goodwin, R.H., Tompkins, G.J., and McCawley, P. (1977). The establishment of two cell lines from the insect Spodoptera frugiperda (Lepidoptera; Noctuidae). In Vitro 13, 213-217.

Vojtech, L., Woo, S., Hughes, S., Levy, C., Ballweber, L., Sauteraud, R.P., Strobl, J., Westerberg, K., Gottardo, R., Tewari, M., et al. (2014). Exosomes in human semen carry a distinctive repertoire of small non-coding RNAs with potential regulatory functions. Nucleic Acids Res 42, 7290-7304.

Wetterwald, C., Roth, T., Kaeslin, M., Annaheim, M., Wespi, G., Heller, M., Maser, P., Roditi, I., Pfister-Wilhelm, R., Bezier, A., et al. (2010). Identification of bracovirus particle proteins and analysis of their transcript levels at the stage of virion formation. J Gen Virol 91, 2610-2619.

Wohlgemuth, I., Brenner, S., Beringer, M., and Rodnina, M.V. (2008). Modulation of the rate of peptidyl transfer on the ribosome by the nature of substrates. J Biol Chem 283, 32229-32235.

Wolff, E.C., Lee, Y.B., Chung, S.I., Folk, J.E., and Park, M.H. (1995). Deoxyhypusine synthase from rat testis: purification and characterization. J Biol Chem 270, 8660-8666.

Xie, Y., Wu, G., Tang, J., Luo, R., Patterson, J., Liu, S., Huang, W., He, G., Gu, S., Li, S., et al. (2014).

SOAPdenovo-Trans: de novo transcriptome assembly with short RNA-Seq reads. Bioinformatics 30,16601666.

Yanase, T., Yasunaga, C., and Kawarabata, T. (1998). Replication of Spodoptera exigua nucleopolyhedrovirus in permissive and non-permissive lepidopteran cell lines. Acta Virol 42, 293-298.

Yu, D.S., Chen, Y.B., Li, M., Yang, M.J., Yang, Y., Hu, J.S., and Luo, K.J. (2016). A polydnaviral genome of Microplitis bicoloratus bracovirus and molecular interactions between the host and virus involved in NFkappaB signaling. Arch Virol 161, 3095-3124.

Zerbino, D.R., and Birney, E. (2008). Velvet: Algorithms for de novo short read assembly using de Bruijn graphs. Genome Research 18, 821-829.

Zhang, H., and Simon, A.K. (2020). Polyamines reverse immune senescence via the translational control of autophagy. Autophagy 16, 181-182.

Zhang, L.D., Cai, Q.C., Cui, J.H., Zhang, W., Dong, S.M., Xiao, W., Li, J., Kou, T.C., Zhang, X.W., He, H.J., et al. (2019). A secreted-Cu/Zn superoxide dismutase from Microplitis bicoloratus reduces reactive oxygen species triggered by symbiotic bracovirus. Dev Comp Immunol 92, 129-139.

Zhang, Y., Wang, X., Yang, H., Liu, H., Lu, Y., Han, L., and Liu, G. (2013). Kinase AKT controls innate immune cell development and function. Immunology 140, 143-152. 


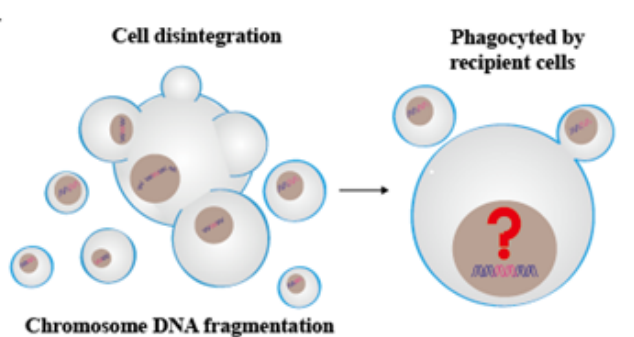

C

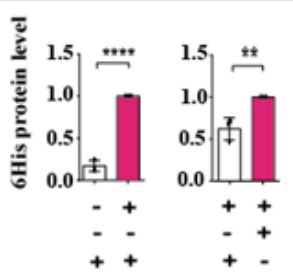

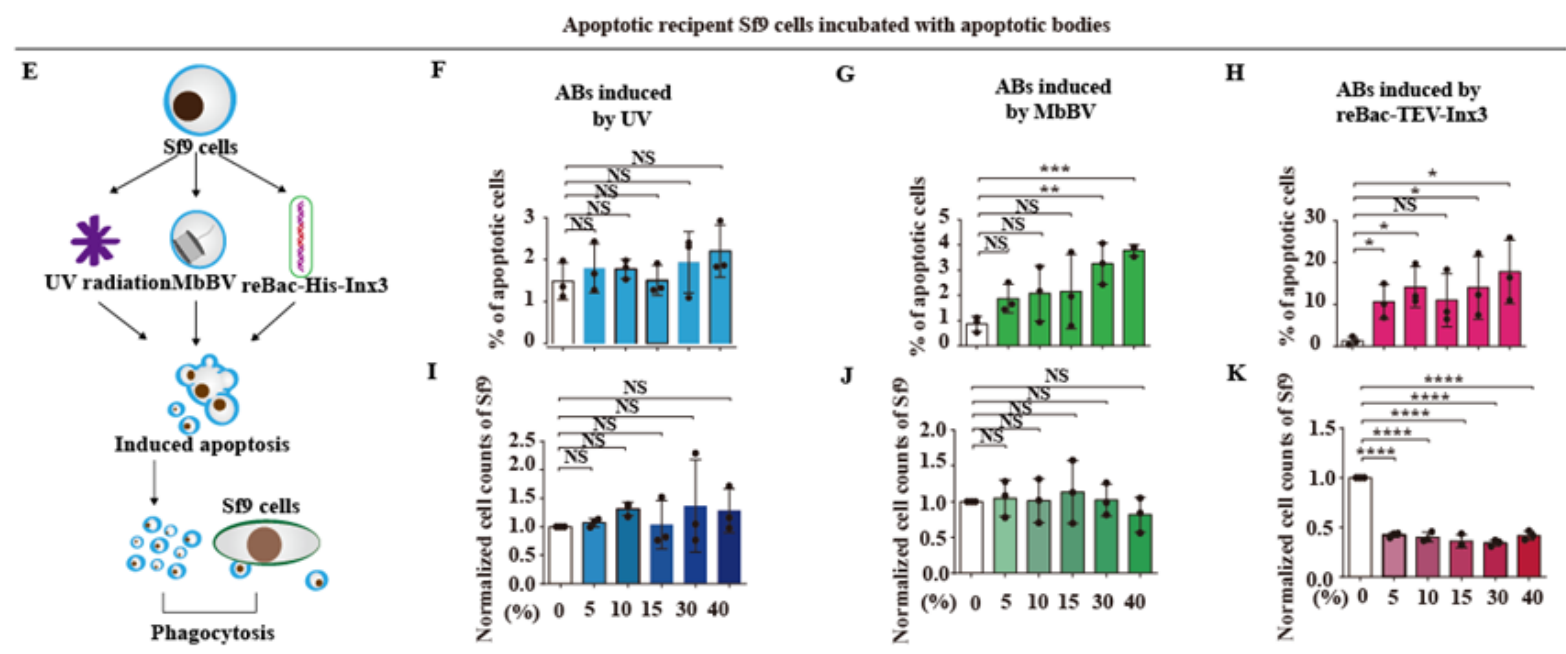

Migration of recipent High Five cells incubated with apoptotic bodies

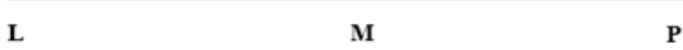

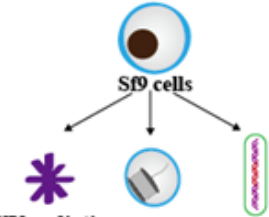

UV radiation $\mathrm{MbBV}$ reBac-His-Inx 3

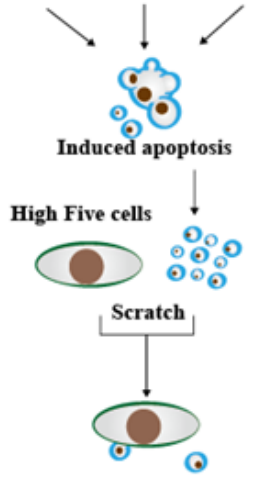

Phagocytosis

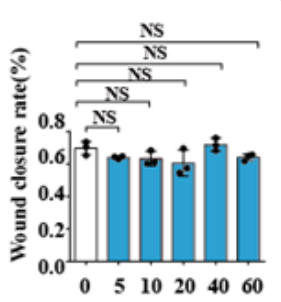

$\mathbf{N}$

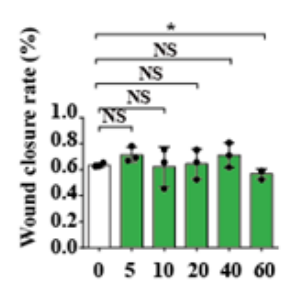

O

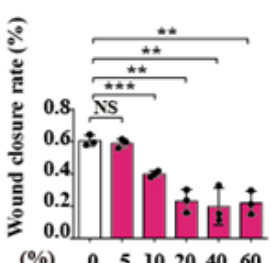

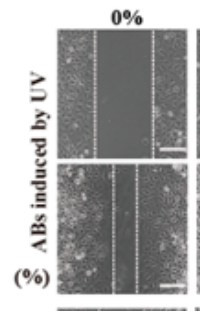
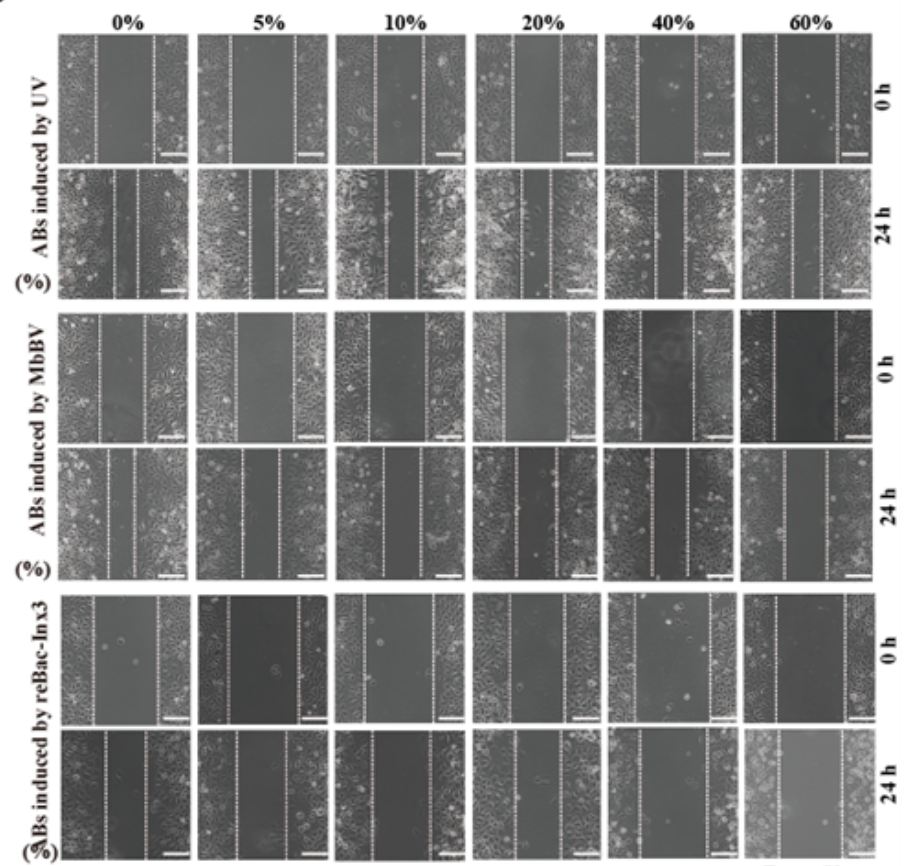

$\underline{m}$

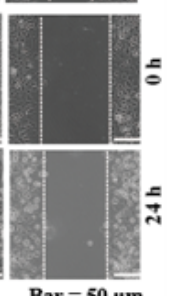

\section{Figure 1}

Bracovirus mediated extracellular vesicles transmit immunosuppressive signaling 
A

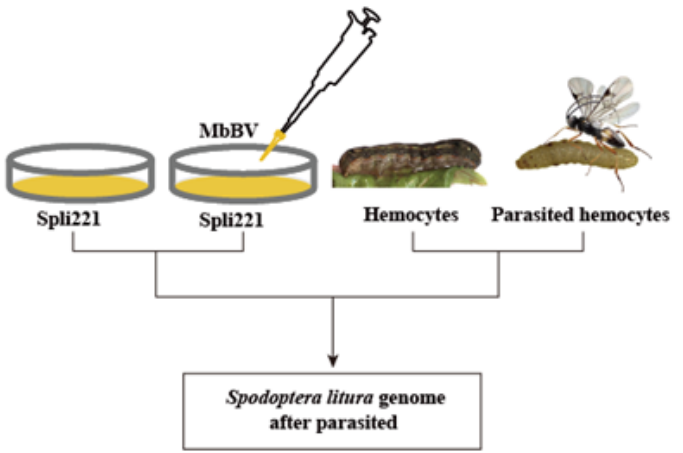

D

Integrated MbBV DNA HIMs in post parasitism hemocytes

B Sequencing scaffold with detected Integration events

Spodoptera litura chromosomes

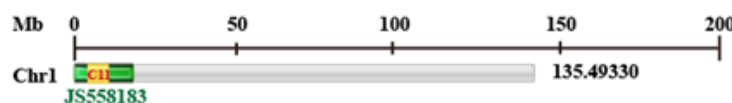

JS558183

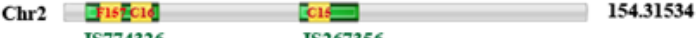

JS774326 JS267356

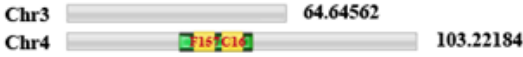

Chr5 118.983534

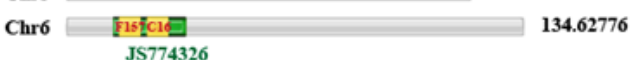

Chr7 प15:c10 154.85697

Chr8 124.21978

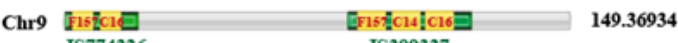
\begin{tabular}{rr} 
JS774326 & J599327 \\
\hline
\end{tabular}

Chr10 Grisicie

Chrll JS774326 133.00942

Chr12 অ15:c19 104.76686

Chr13 $\quad$ JS774326 73.52807

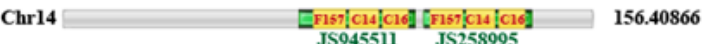
Chr15 E틸 139.26079 JS441032 JS774326 JS774326 162.71522

Chr17 Ecil] G G15icld] 135.92034 JS726283 JS774326

Chr18 $\quad$ [-F157]c14] 121.91718

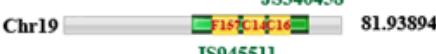

Chr20 $\quad$ JS945511 136.28927 JS945511

Chr21 142.08890

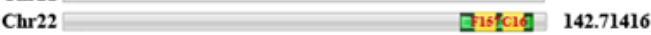

Chr23 $\begin{array}{ll}\text { JS774326 } \\ 138.80142\end{array}$

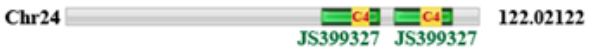

Chr25 דisic1] 136.83143

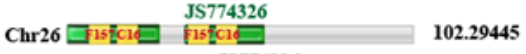

JS774326 JS774326

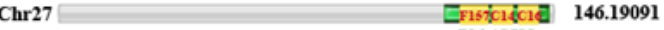
JS945511

\begin{tabular}{|c|c|}
\hline & JS945511 \\
\hline & \\
\hline Q715:ciel] & 142.13186 \\
\hline JS774326 & $\begin{array}{r}\text { JS511984 } \\
130.13156\end{array}$ \\
\hline
\end{tabular}

Chr30

ChrZ

189.34769

\section{B}

MbBVDNA Junction 1 Host Chr (29 bp)TGCGATOCTAGTAGCAAATGACCGGGACCCTGTGG(84 bp) JS258995 (122 bp) (21 bP)AGCACTTCTAGA LAGGIATAAGGGTATAATAATATTI(84 bp) JS267356 (123 bp) (26 bP)ATCACTACTAGT RACATTGCTGGTACGATCTTGATT(104 bp) JS399327 (139bp) (30 bp)TGCATTACAAG CAAAGTTTAACTTIATCTAAACA(113 bp) JS558183 (150 bp) (31 bP)IGCGATOCTAGI CCGATATCAACATGAAATCAACAT(123 bp) JS945511 (265 bp) (109 bP)TTATTATT GACAACTGTIOCTAGACATGCAGGTACA(37 bp) JS511984 (146 bp)

C

Host Chr Junction 2 MbBV DNA ( 80 bp)ATTIAATTAATAAGTIATTTTIAATT ACTAGAAGTGCT(21 bD) JS198167 (115bp) (99 bp) GCATCGAAACATTAAATCGTAATA ACCAGGATTGAA2I bP) JS340458 (132 bp) (107 bp) GTTIAACATTATGTATTGGAATTG ACTAGGACTGAT(29 bD JS441932 (142 bp) (129 bP)TLATGAGTTTTTGTATAAAAATAT CTAGGGATIATT (29 bD) JS726283 (161 bp) (74 bP) TCTGAATCTGTTCACAGTAACTAG ACTAGGATCGCA(29 bD JS774326 (174 bp) (440 bp)IC GGTTIAGTTGTTCTCGAGTTA ACTAGGATC CCA30 bD) JS1070115 (474 bp)

$\mathbf{E}$ Chrl $150 \mathrm{bp}$

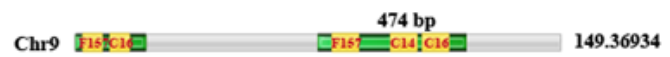

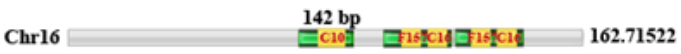

Chr19 $\square \stackrel{265 \text { bp }}{\square} 81.93894$

F

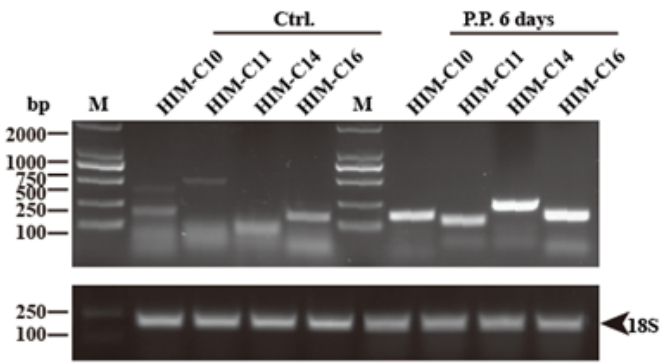

G

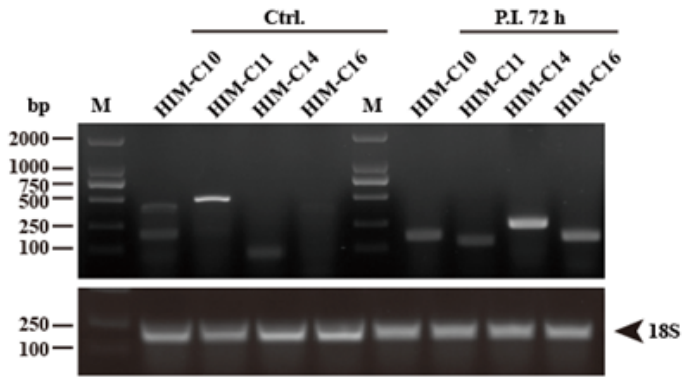

H

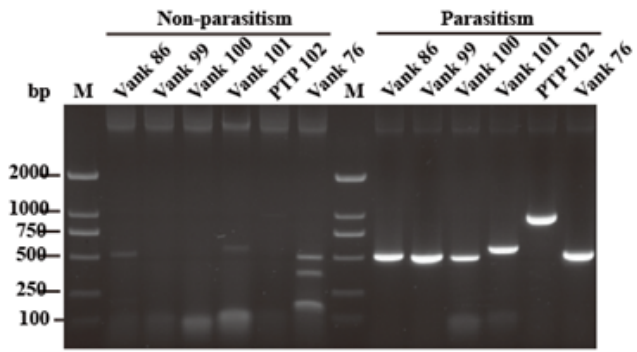

Figure 2

Bracovirus primary integrates via HIM sites 
A

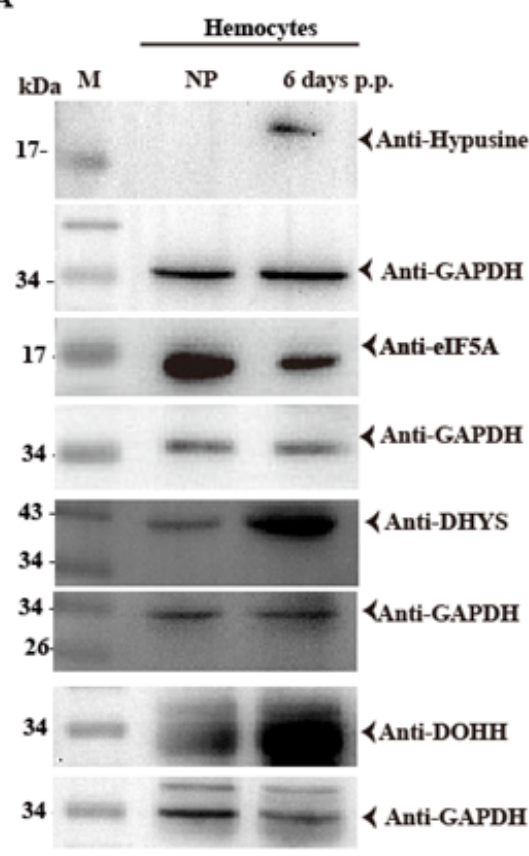

C

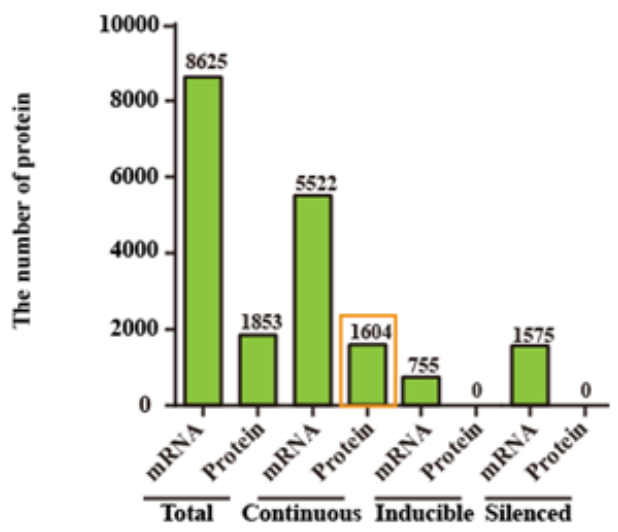

G

\begin{tabular}{|c|c|c|}
\hline \multicolumn{3}{|c|}{ Spodoptera litura eIF5A } \\
\hline \multicolumn{3}{|c|}{ K52 } \\
\hline DHYS ¿ & & \\
\hline 1 & & 160 \\
\hline Spodoptera litura & 45 M S T K T GKH G H A K V H 59 & (K52) \\
\hline Spodoptera frugiperda & 45 M S T S K T G K H G H A K V H 59 & (K52) \\
\hline $\begin{array}{l}\text { Homo sapiens } \\
\text { Mus musculus }\end{array}$ & $\begin{array}{l}\text { 43 M ST ST GKH GHAKVH } 57 \\
\text { 43M } \text { M S T GKH GHAKVH } 57\end{array}$ & $\begin{array}{l}\text { (K50) } \\
\text { (K50) }\end{array}$ \\
\hline Danio rerio & 44 M S T S K T G K H G H A K V H 58 & (K51) \\
\hline izosaccharomyces pomb & 57 M S T S K T G KH G H A K V H 71 & (K64) \\
\hline
\end{tabular}

\section{Figure 3}

Bracovirus integration triggered elF5A hypusination

$\mathbf{E}$

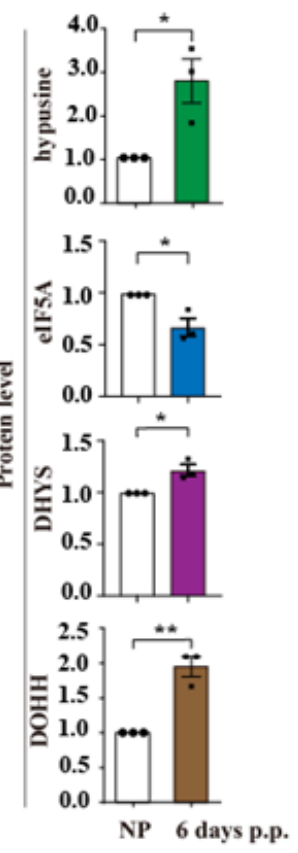

D

H

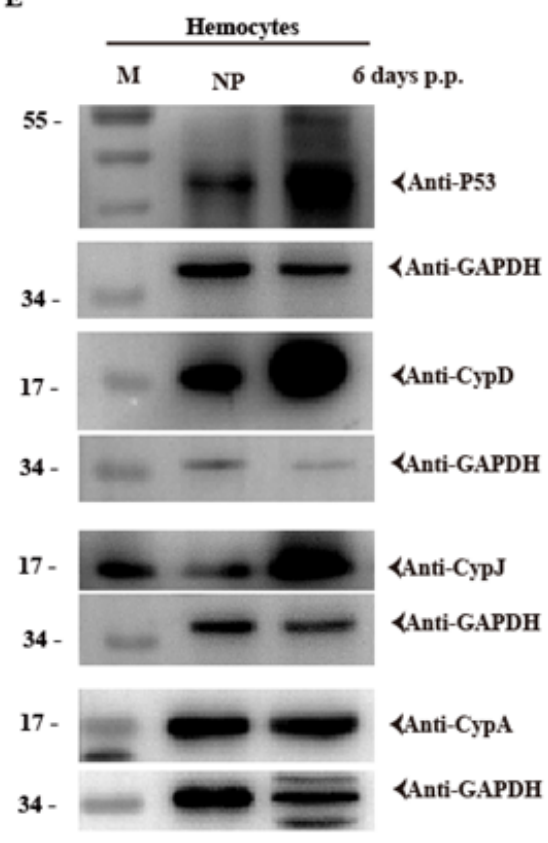

F
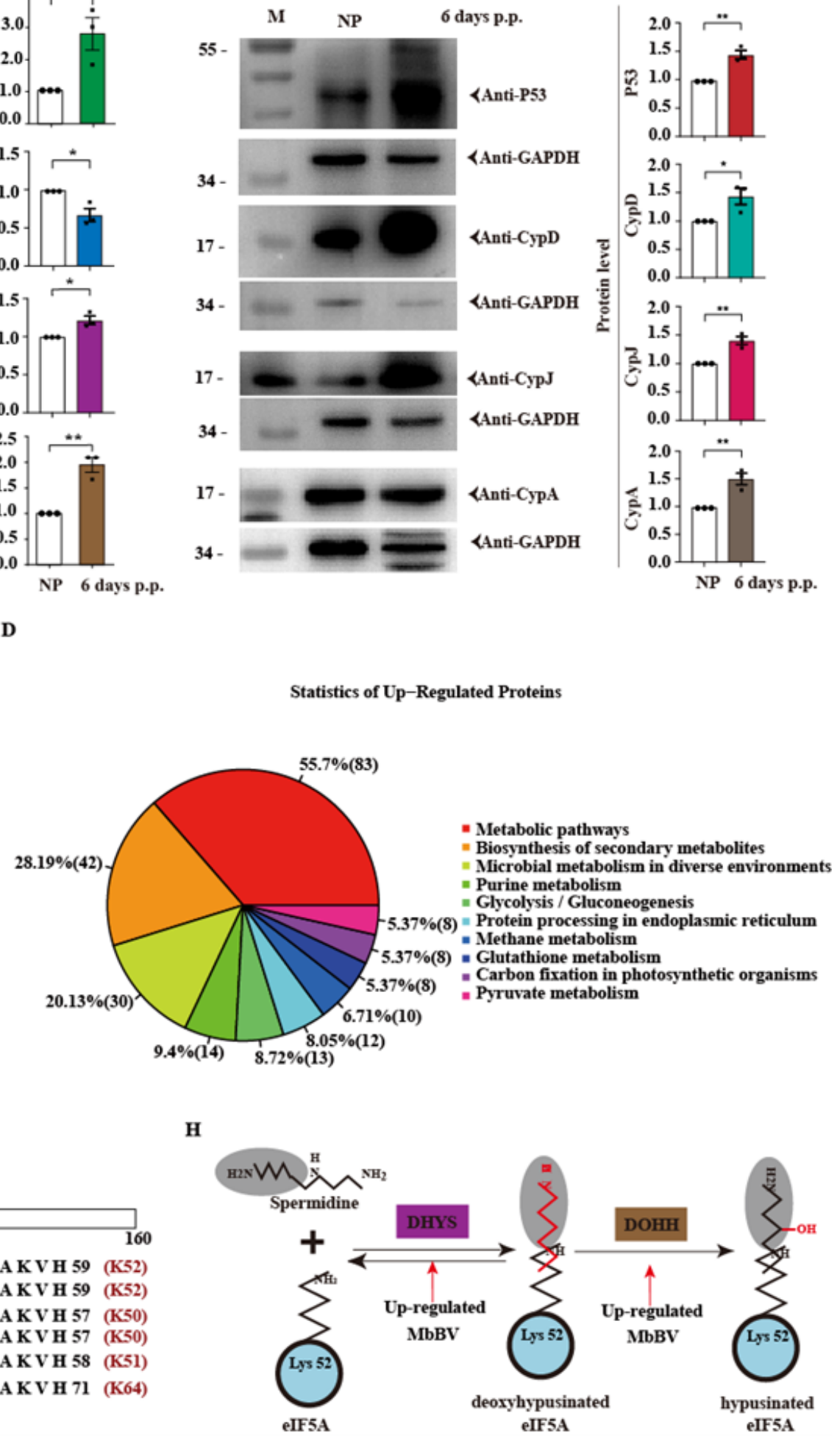
A
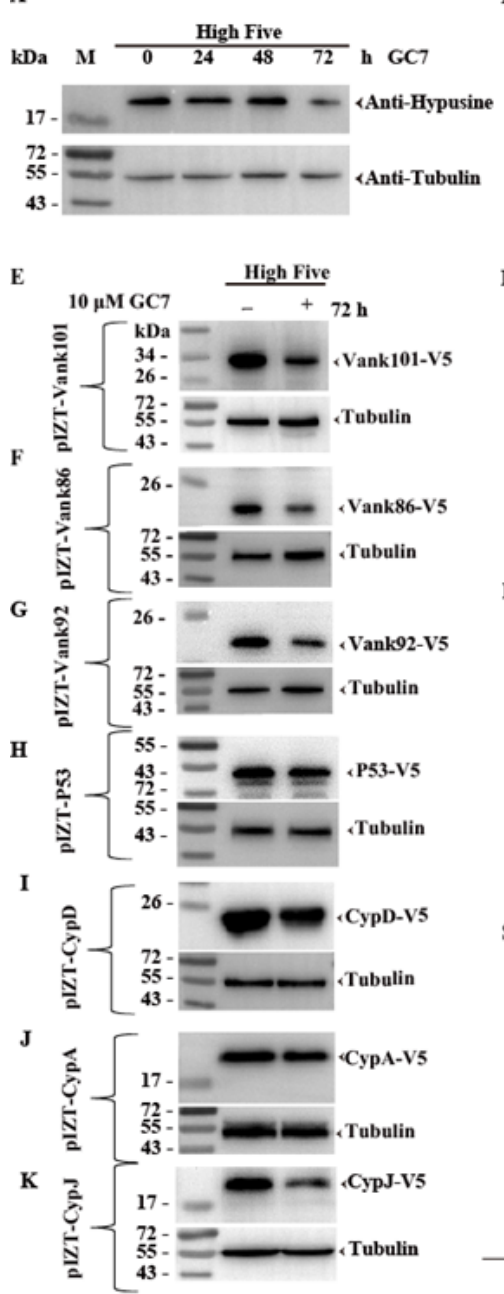

W
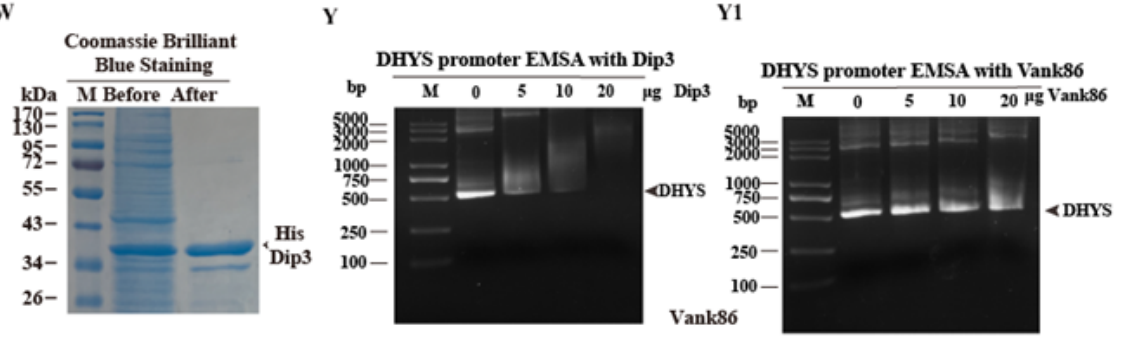

X $\begin{gathered}\text { Coomassie Brilliant } \\ \text { Blue Staining }\end{gathered}$

kDa M Before After

$130=$

95-

72-

55-

$34-$

26-

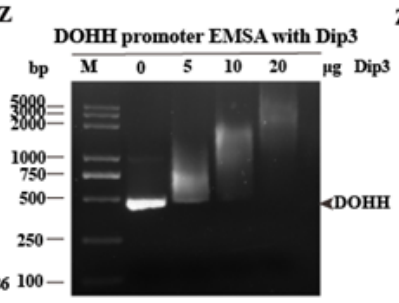

C

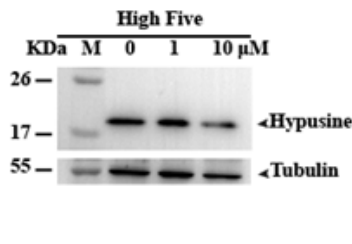

L
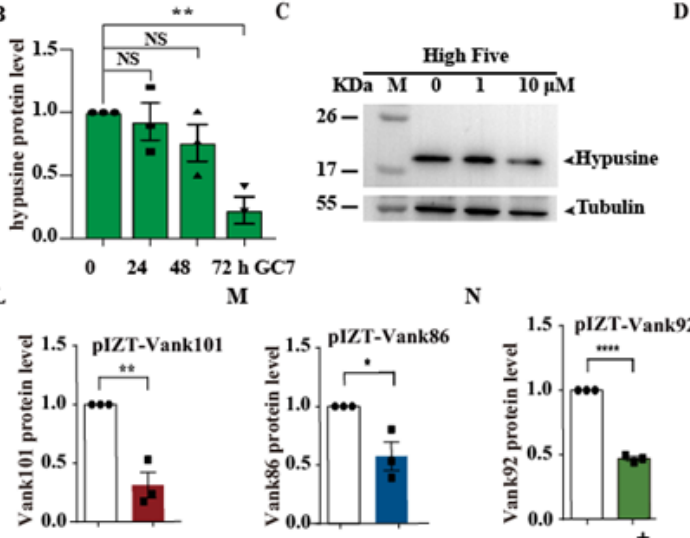

$10 \mu \mathrm{M} \mathrm{GC7}(72 \mathrm{~h})$
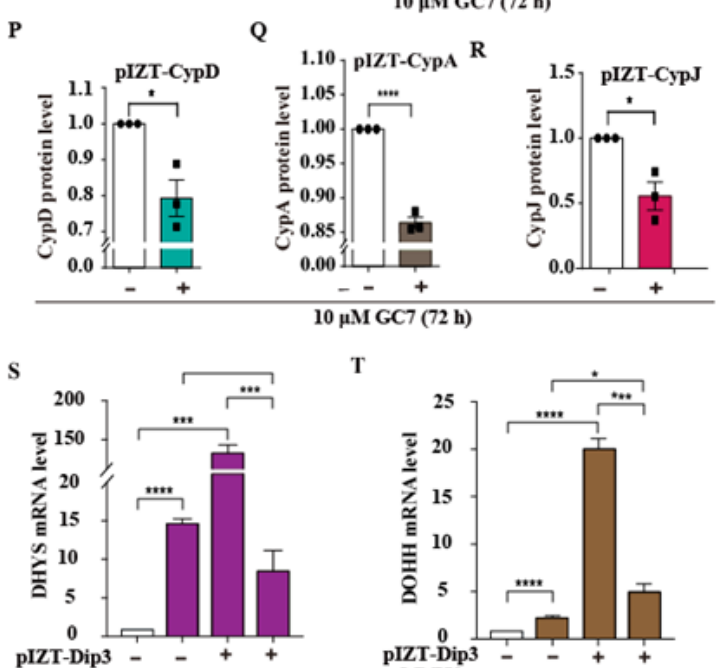

$\mathbf{T}$
$10 \mu \mathrm{M} \mathrm{GC7}$ (72 h)

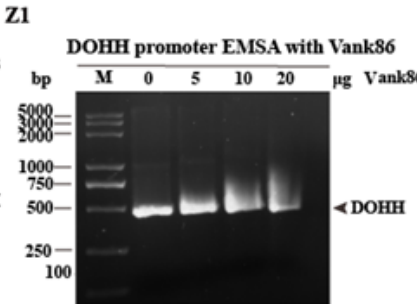

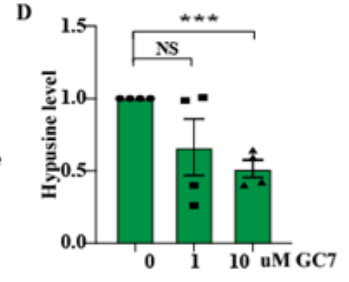
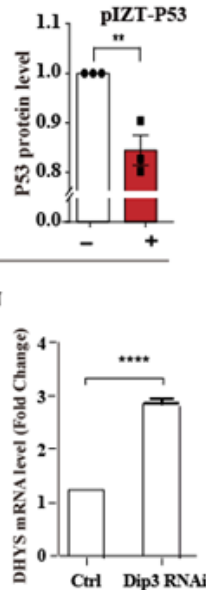

हัّ.

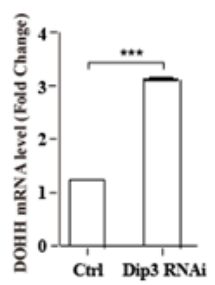

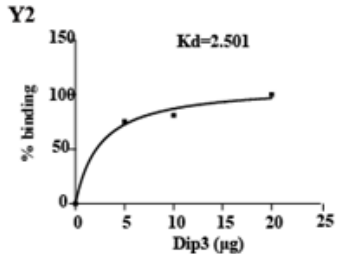

5 ' IATTCCCATTTTCAGTCC GGCATTAACTGATG 3. DHYS promoter bingding site

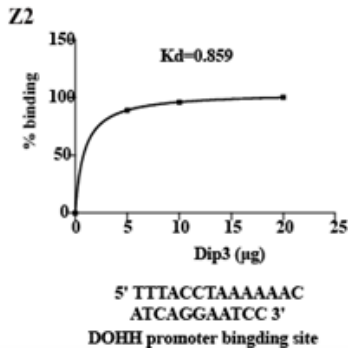

Figure 4

Bracovirus integrated viral genes dependent elF5A hypusination translation pattern and viral proteins positively regulated DHYS and DOHH expression 
A

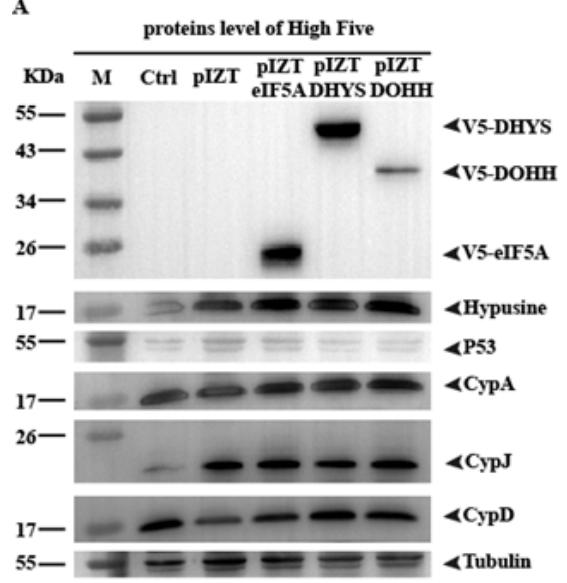

F

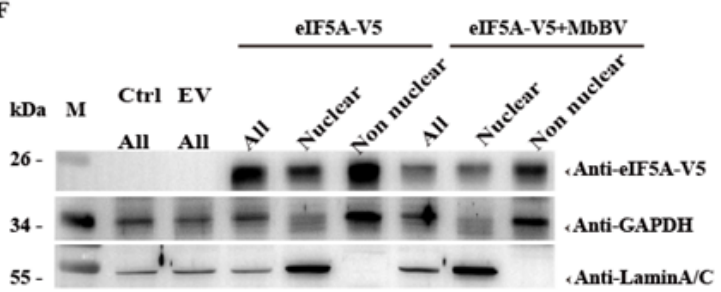

B
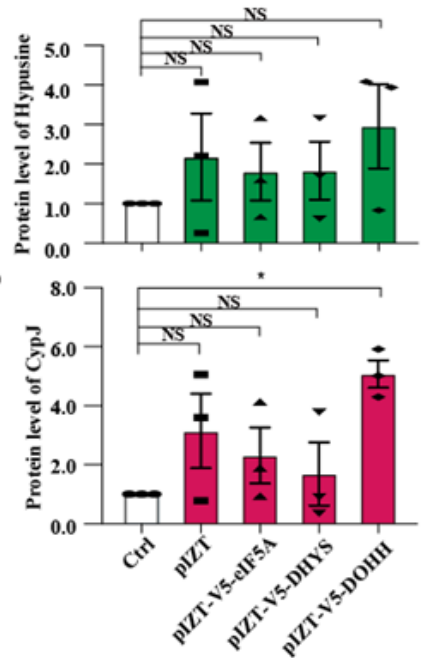

G

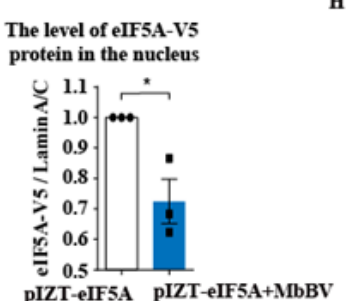

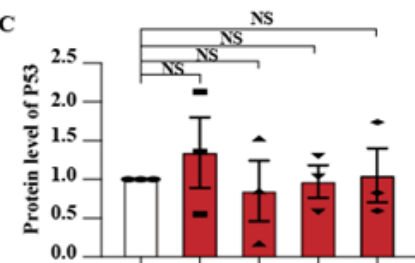

E

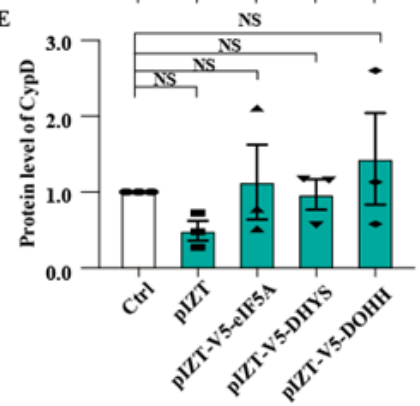

H

The level of eIF5A-V5 protein in the cytoplasm

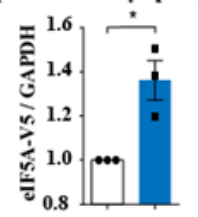

pIZT-eIF5A pIZT-eIF5A+MbBV

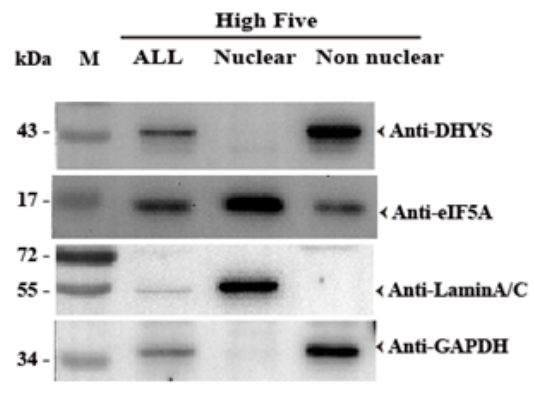

K

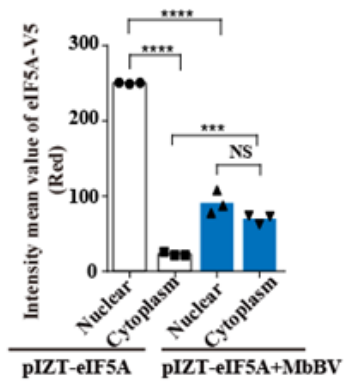

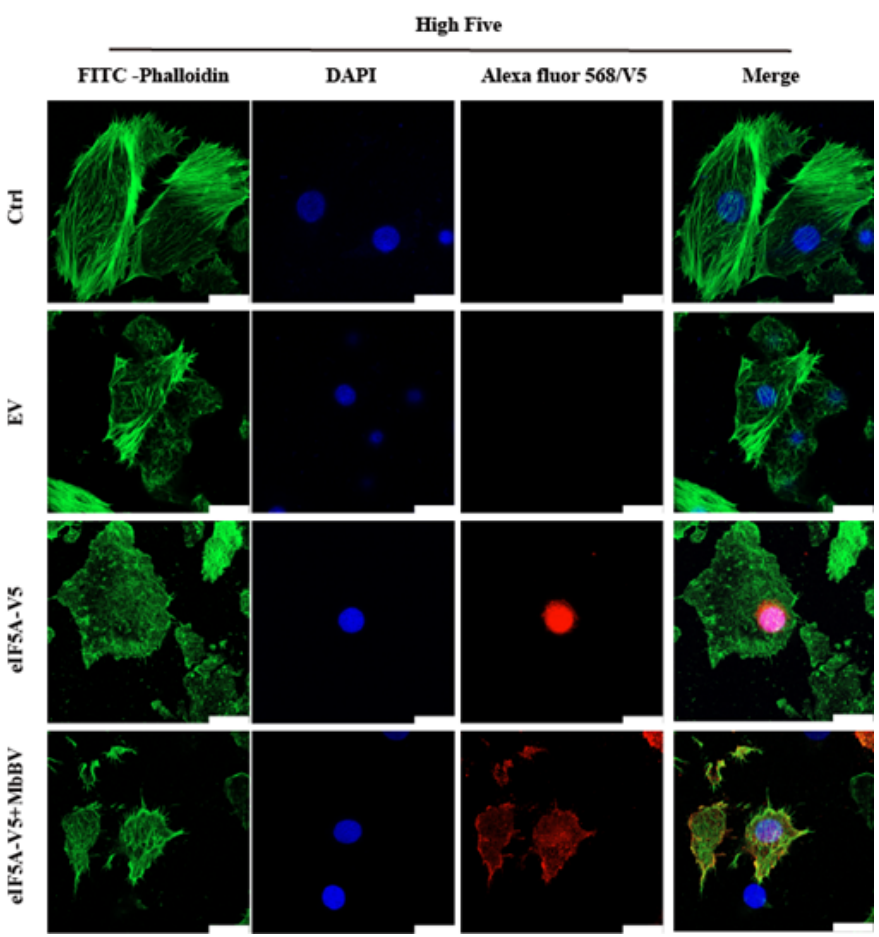

\section{Figure 5}

Bracovirus activated hypusination via elF5A nuclear-cytoplasmic transport 

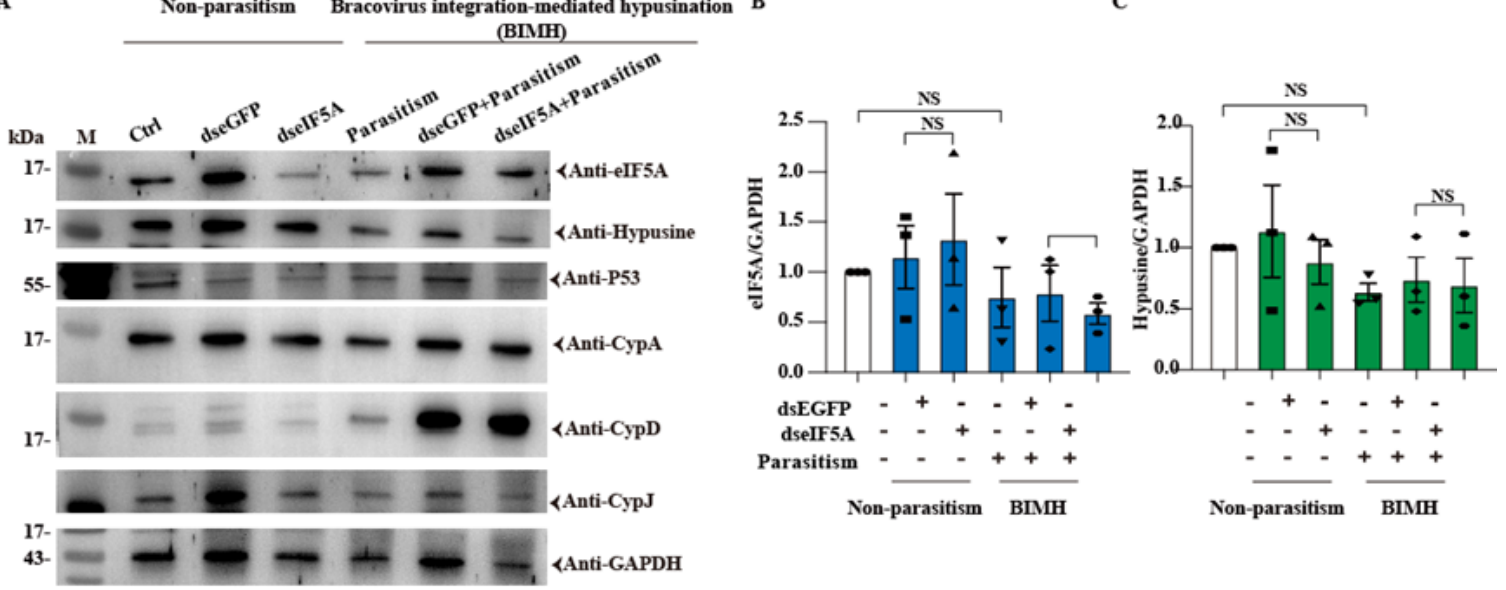

D
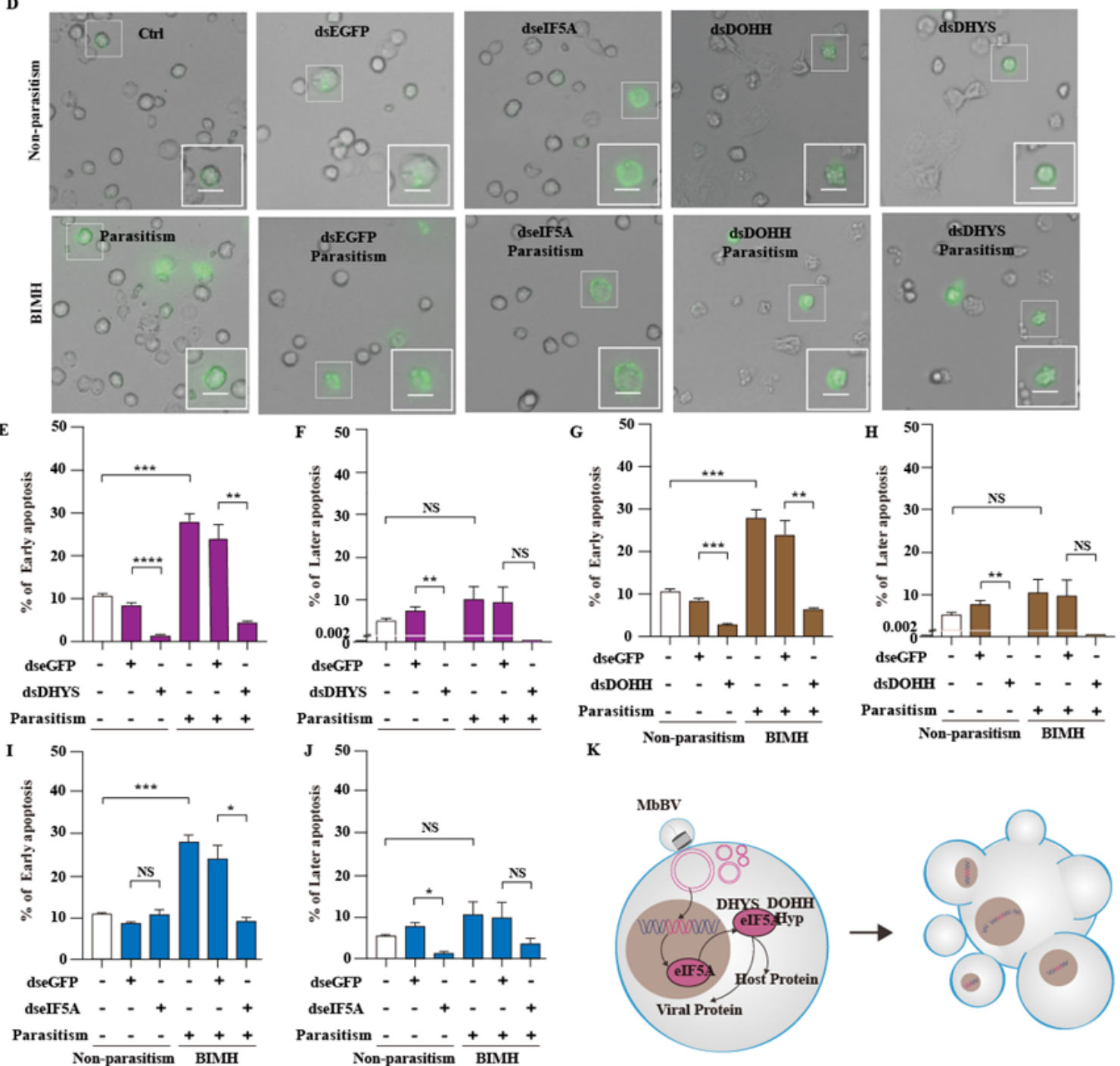

K

Non-parasitism BIMH

\section{Figure 6}

Bracovirus integration-mediated hypusination drives persistence apoptosis 


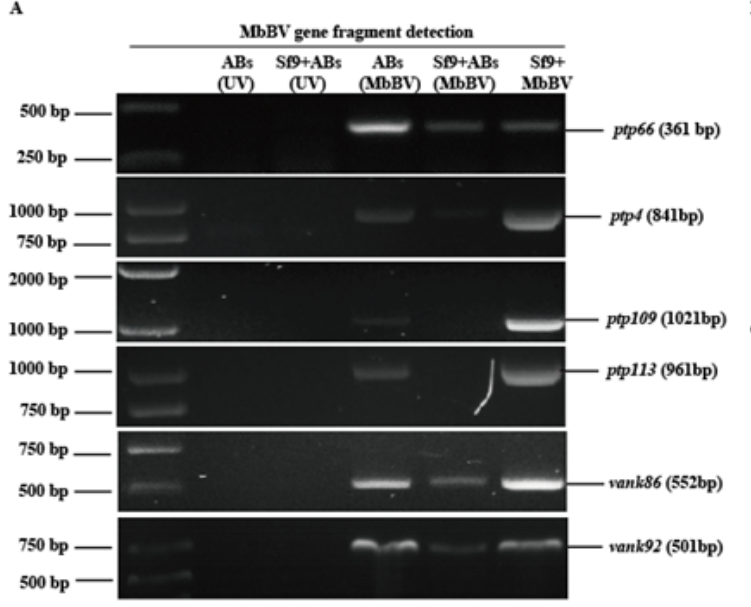

D
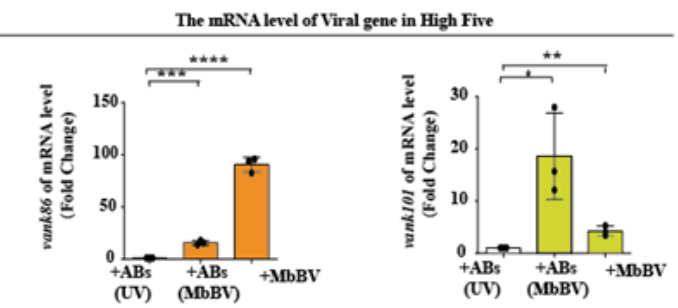

$\mathbf{E}$
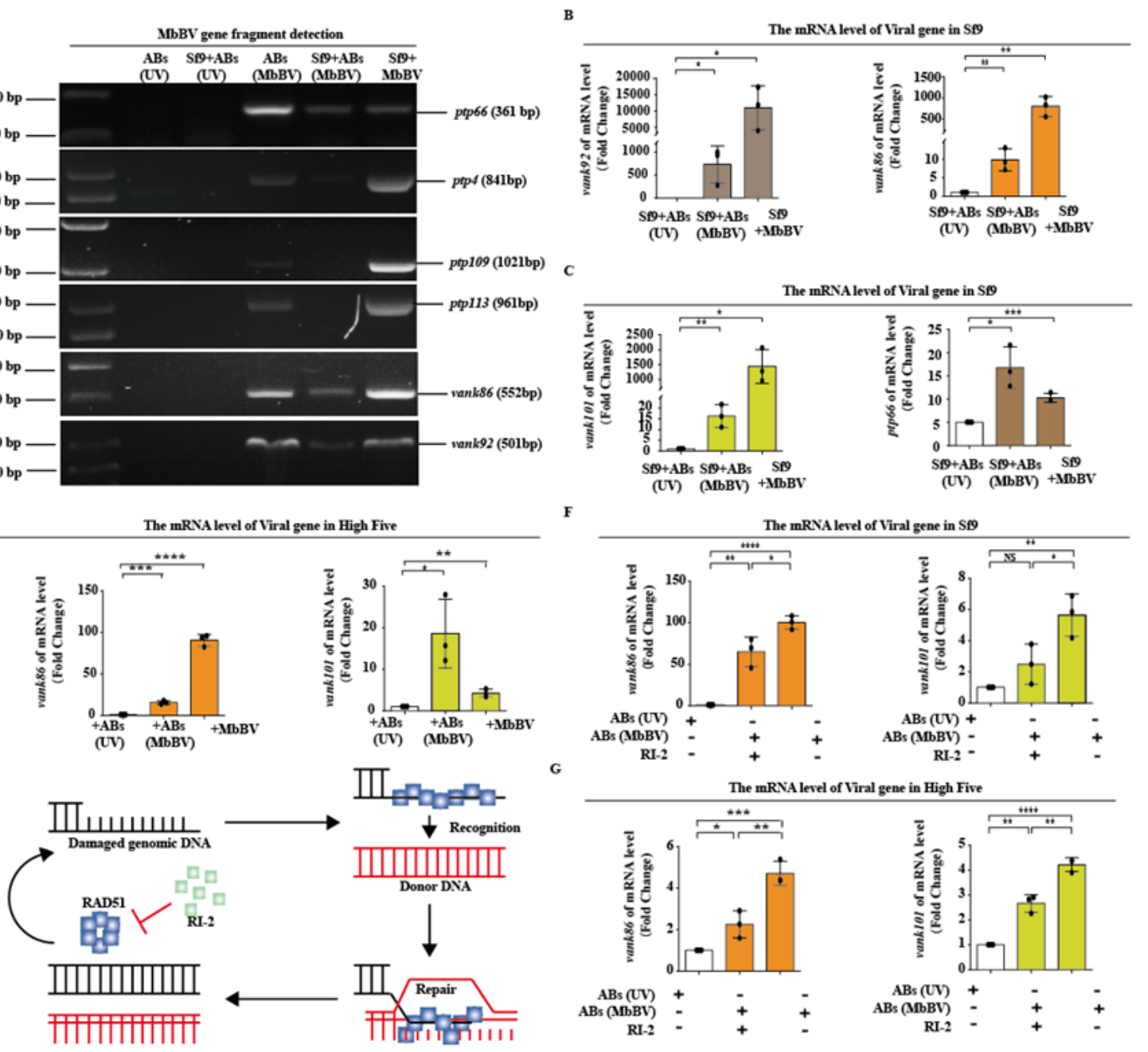

$\mathbf{F}$
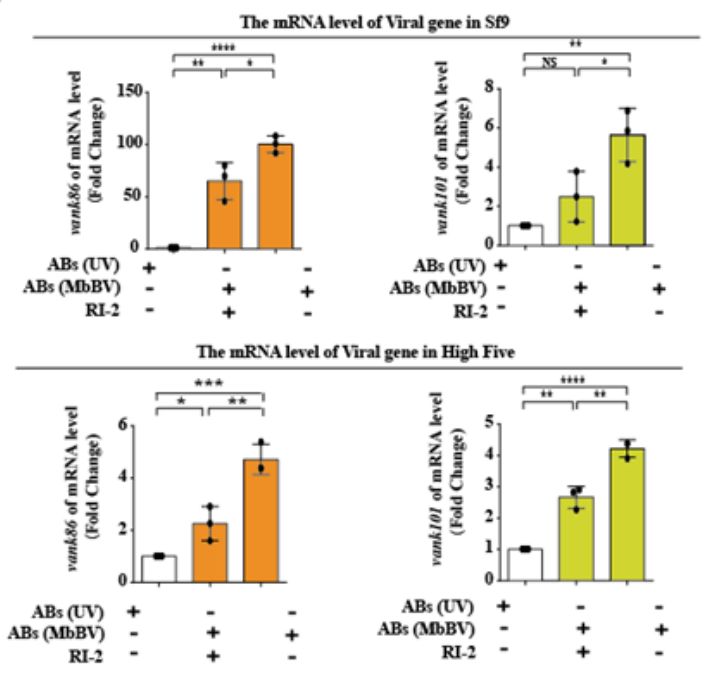

The recipient cells of SPF

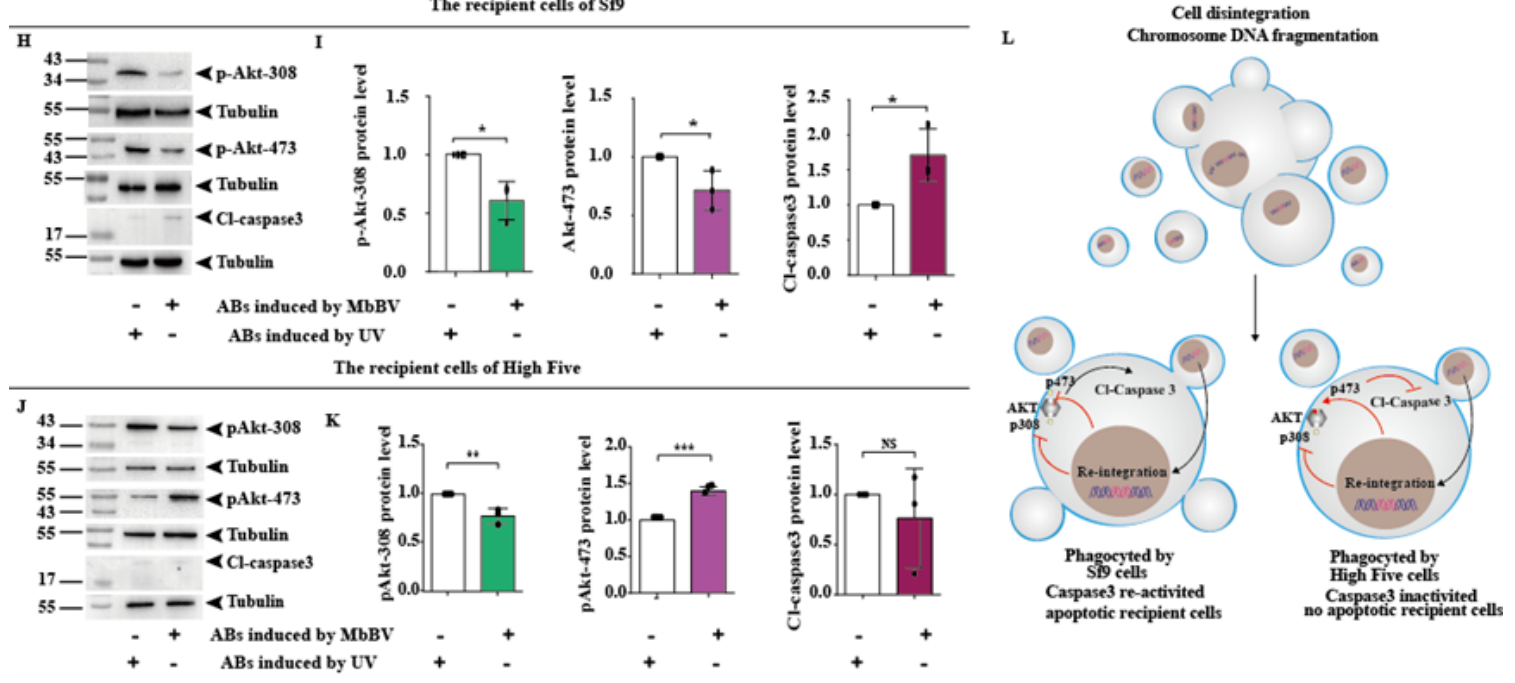

Figure 7

Bracovirus fragments in extracellular vesicles re-integrate into recipient cells

\section{Supplementary Files}

This is a list of supplementary files associated with this preprint. Click to download. 
- 20211104SupplementallnformationBracovirussneaksinto.docx 